\title{
Chemical Topology of Crystalline Matter and the Transcendental Numbers $\phi$, e and $\pi$
}

\author{
Michael J. Bucknum ${ }^{\beta}$ \& Eduardo A. Castro \\ INIFTA, Theoretical Chemistry Division, Universidad de La Plata, La Plata 1900, Buenos \\ Aires, Argentina
}

\begin{abstract}
In this paper, we describe the tenets of a chemical topology of crystalline matter and certain associated rational approximations to the transcendental mathematical constants $\phi$, e and $\pi$, that arise out of considerations of both: (1) the Euler relation for the division of the sphere into vertices, V, faces, F, and edges, E, and: (2) its simple algebraic transformation into the so-called Schläfli relation, which is an equivalent mathematical statement for the polyhedra, in terms of parameters known as the polygonality, defined as $\mathrm{n}=2 \mathrm{E} / \mathrm{F}$, and the connectivivty, defined as $\mathrm{p}=2 \mathrm{E} / \mathrm{V}$. It is thus the transformation to the Schläfli relation from the Euler relation, in particular, that enables one to move from a simple heuristic mapping of the polyhedra in the space of $\mathrm{V}, \mathrm{F}$ and $\mathrm{E}$, into a corresponding heuristic mapping into Schläfli-space, the space circumscribed by the parameters of $\mathrm{n}$ and p. It is also true, that this latter transformation equation, the Schläfli relation, applies only directly to the polyhedra, again, with their corresponding Schläfli symbols (n, p), but as a bonus, there is a direct 1-to-1 mapping result for the polyhedra, that can be seen to also be extendable to the tessellations in 2-dimensions, and the networks in 3-dimensions, in terms of coordinates in a 2-dimensional Cartesian grid, represented as the Schläfli symbols (n, p), as discussed above, which do not involve rigorous solutions to the Schläfli relation. For while one could never identify the triplet set of integers (V, F, E) for the tessellations and networks, that would fit as a rational solution within the Euler relation, it is in fact possible for one to identify the corresponding values of the ordered pair (n, p) for any tessellation or network. The identification of the Schläfli symbol (n, p) for the tessellations and networks emerges from the formulation of its so-called Well's point symbol, through the proper translation of that Well's point symbol into an equivalent and unambiguous Schläfli symbol (n, p) for a given tessellation or network, as has been shown by Bucknum et al. previously. What we report in this communication, are the computations of some, certain Schläfli symbols ( $\mathrm{n}, \mathrm{p}$ ) for the so-called Waserite (also called platinate, $\mathrm{Pt}_{3} \mathrm{O}_{4}$, a 3,4-connected cubic pattern), Moravia $\left(\mathrm{A}_{3} \mathrm{~B}_{8}\right.$, a 3-,8-connected cubic pattern) and Kentuckia ( $\mathrm{ABC}_{2}$, a 4-,6-,8-connected tetragonal pattern) networks, and some topological descriptors of other relevant structures. It is thus seen, that the computations of the polygonality and connectivity indexes, $\mathrm{n}$ and $\mathrm{p}$, that are found as a consequence of identifying the Schläfli symbols for these relatively simple networks, lead to simple and direct connections to certain rational approximations to the transcendental mathematical constants $\phi$, e and $\pi$, that, to the author's knowledge, have not been identified previously. Such rational approximations lead to elementary and straightforward methods to estimate these mathematical constants to an accuracy of better than 99 parts in 100 .
\end{abstract}

\footnotetext{
${ }^{\beta}$ corresponding author, mjbucknum@gmail.com
} 


\section{Introduction}

Bucknum [1] in work first described in 1997, outlined a general scheme for the systematic classification and mapping of the polyhedra, 2-dimensional tessellations and 3-dimensional networks in a self-consistent topological space for these structures. This general scheme begins with a consideration of the Euler relation [2] for the polyhedra, shown below as Equation (1), which was first proposed in 1758 to the Russian Academy by Euler, and was, in fact, the point of departure for Euler into a new area of mathematics thereafter known explicitly as topology.

$$
\mathrm{V}-\mathrm{E}+\mathrm{F}=2
$$

Relation (1) stipulates that for any of the innumerable polyhedra, the combination of the number of vertices, $\mathrm{V}$, minus the number of edges, $\mathrm{E}$, plus the number of faces, F, resulting from any such division of the sphere, will invariantly be that number 2, known as the Euler characteristic of the sphere. The variables known as $\mathrm{V}, \mathrm{E}$ and $\mathrm{F}$ are topological properties of the polyhedra, or, in other words, they are invariants of the polyhedra under any kind of geometrical distortions. It is from this simple Eulerian relation, that we can develop a systematic and, indeed otherwise rigorous, mapping of the various, innumerable structures that present themselves, in levels of approximation, as models for the structure of the real material world within the domain of that area of science known as crystallography.

About a century after Euler's relation for the polyhedra was first proposed, as described above, the German mathematician Schläfli introduced a simple algebraic transformation of Euler's relation, for various purposes of understanding the relation better, and adopting it more effectively in proofs 
[3]. Thus, Schläfli introduced two new topological variables, like V, E and F before them, that were derived from them. Schläfli, therefore, defined the socalled "polygonality", hereafter represented by n, of a polyhedron as the averaged number of sides, or edges, circumscribing the faces of a polyhedron. He conveniently defined such a polygonality, as $n=2 E / F$, where, in this instance one can see that because each edge E straddles two faces, F, the definition is rigorous. Similarly, Schläfli introduced the topological parameter called the "connectivity", hereafter represented by $\mathrm{p}$, of a polyhedron as the averaged number of sides, or edges, terminating at each vertex of a polyhedron. He conveniently defined such a connectivity, as $\mathrm{p}=2 \mathrm{E} / \mathrm{V}$, where, in this instance one can see that because each edge $E$ terminates at two vertices, $\mathrm{V}$, the definition is rigorous.

From these definitions of $\mathrm{n}$ and $\mathrm{p}$ as topological parameters of the polyhedra, Schläfli was able to show quite straightforwardly, by algebraic substitution, that a further relation exists among the polyhedra in terms of their Schläfli symbols (n, p). [3] It is from this equation, the Schläfli relation, shown as Equation (2) below, that one can see that not only do the polyhedra rigorously obey 2 , but it is also true that their indices as (n, p), that serve as solutions to 2, in addition lead to a convenient 2-dimensional grid, or Schläfli space, over which the various polyhedra can be unambiguously mapped, as has been explained by Wells in his important 1977 monograph on the subject [4].

$$
\frac{1}{n}-\frac{1}{2}+\frac{1}{p}=\frac{1}{E}
$$


Figure 1 due to Wells [4], below, illustrates the application of this type of Schläfli mapping for the regular Platonic polyhedra, where one sees that the point that belongs to the origin of this mapping, is indeed given by the Schläfli symbol $(n, p)=(3,3)$. The symbol $(3,3)$ represents the Platonic solid known as the tetrahedron, or by the symbol " $\mathrm{t}$ " in the map, known since Antiquity by the Greeks. Thus, as it is cast as the origin of this mapping of polyhedra, it is apparently, the only self-dual polyhedron. Similarly $(4,3)$ is the Platonic solid of the Greeks known as the cube, or "c" in the map, $(5,3)$ is the Platonic solid of the Greeks known as the dodecahedron, or " $\mathrm{d}$ " in the map, $(3,4)$ is the Platonic solid of the Greeks known as the octahedron, or " $\mathrm{o}$ " in the map, and $(3,5)$ is the Platonic solid of the Greeks known as the icosahedron, or "i" in the map.

\section{Figure 1}

Although the mapping of the Platonic polyhedra, shown in Figure 1, indeed involves only those polyhedra in which the ordered pairs (n, p) are integers, it is readily transparent that one could magnify the map to include those polyhedra in which the polygonality " $n$ " is fractional, these are the socalled Archimedean polyhedra, discovered by Archimedes in Ancient Greece [5]. In addition, the map could be magnified to include those polyhedra in which the connectivity " $p$ " is fractional, these are the so-called Catalan polyhedra, discovered in Europe in the $19^{\text {th }}$ century [6]. And finally, the socalled Wellean polyhedra [7], discovered in the $21^{\text {st }}$ century [8], in which both indexes, "n" and "p", are fractional, could be mapped in this Schläfli space of the polyhedra, without any loss of mathematical rigor. 


\section{Mapping of Tessellations \& Networks in Schläfli Space}

Wells also suggested [4], that the tessellations, which are structures or tilings extended into 2-dimensions and filling the plane, could have nominal labels attached to them, in the form of the Schläfli symbols (n, p), that, while not leading to rational solutions of the Schläfli relation, were, still it seems, rigorously defined from inspection of the topology of these elementary tessellations. Thus in order to extend the mapping in Schläfli space, the space of (n, p), some loss of rigor with regard to the Schläfli relation for the polyhedra had to be introduced, when considering the patterns known as tessellations. Wells, therefore included, explicity, the mapping of the square grid, given by the Schläfli symbol $(4,4)$, and the honeycomb grid, given by $(6,3)$, as well as the closest-packed tessellation, given by $(3,6)$, in his topology mapping, shown in Figure 1, along with the Platonic polyhedra. He thereby extended the mapping to the tessellations, and later he implied that such a mapping could be extended to include the 3-dimensional (3D) networks as well, with a concomitant further loss of mathematical rigor, in that the values assigned as (n, p) to the various tessellations and networks were not rigorous solutions to Equation (2).

It is also true that Wells [7], perfectly well introduced a systematic and rigorous coding of the topology of tessellations and networks he worked with, which is now called the Wells point symbol notation, and that this was a simple coding scheme over the circuitry and valences, about the vertices, in the unit of pattern of the tessellations and networks. The Wells point symbol notation was, however, nonetheless an important development for the rigorous mathematical basis it put the tessellations and networks on, formally, as quasi-solutions (n, p) for the Schläfli relation shown as Equation (2). 
Therefore, in a generic case of the Wells point symbol notation, one could have such a symbol for an a-, b-connected, binary network, given as $\left(A^{a}\right)_{X}\left(B^{b}\right)_{Y}$, such that the exponents "a" and " $b$ " nominally represent the valences of the 2 vertices in the tessellation or network of interest, and the bases " $A$ " and "B" give the respective relative polygon sizes (circuit sizes) in the tessellation or network, while the parameters " $\mathrm{X}$ " and "Y" describe the binary stoichiometry of the network. In this generic case, we see that " $\mathrm{X} / \mathrm{Y}$ " represents the number of structural components, identified by their topology character as $\left(\mathrm{A}^{\mathrm{a}}\right)$, to the number of structural components identified by their respective topology as $\left(\mathrm{B}^{\mathrm{b}}\right)$, that occur in this characteristic ratio in the structure, as specified by the unit of pattern.

Despite his invention of this elegant notation, Wells, for some odd reason, never explicitly showed how to translate the language of the Wells point symbol $\left(\mathrm{A}^{\mathrm{a}}\right)_{X}\left(\mathrm{~B}^{\mathrm{b}}\right)_{Y}$, rigorously into a Schläfli symbol $(n, p)$, as was later shown elsewhere. Thus Bucknum et al,, in 2004 [9], showed that the translation of the Wells point symbol into an, otherwise, from the perspective of Equation (2), rigorous set of values (n, p) for the purpose of mapping tessellations and networks, was achievable if one used the following straightforward, simple formulas (applicable in this case for a generic Wellsean, binary stoichiometry structure) for the ordered pair (n, p), which can later be employed in the mapping of the structure, as is shown by Equations (3).

$$
\begin{gathered}
\mathrm{n}=(\mathrm{a} \cdot \mathrm{A} \cdot \mathrm{X}+\mathrm{b} \cdot \mathrm{B} \cdot \mathrm{Y}) /(\mathrm{a} \cdot \mathrm{X}+\mathrm{b} \cdot \mathrm{Y}) \\
\mathrm{p}=(\mathrm{a} \cdot \mathrm{X}+\mathrm{b} \cdot \mathrm{Y}) /(\mathrm{X}+\mathrm{Y})
\end{gathered}
$$


It should, of course, be noted that one has to proceed with care in using Equations ( $3 \mathrm{a} \& 3 \mathrm{~b})$, in this topology analysis of structures, by carefully normalizing the circuitry traced around the p-connected vertices of the structure (paying careful attention to the parameters " $a$ " and " $b$ " above), by rigorously translating the circuitry of a given structure, into a vertex connectivity for the network of interest, by employing a vertex translation table like that shown below.

\section{Table 1}

Therefore, from the use of Equations (3), for a binary stoichiometry, Wellsean net with topology $\left(\mathrm{A}^{\mathrm{a}}\right)_{\mathrm{X}}\left(\mathrm{B}^{\mathrm{b}}\right)_{\mathrm{Y}}$, or some other homologous translation formulas, as shown, for example, by Bucknum et al. for various elementary structural cases [9], it becomes, evidently, rigorously possible to precisely map the topology of any structure, including of course the polyhedra, but extendable to the vast body of the known tessellations and networks, that have been discovered and characterized crystallographically, otherwise by their symmetry character, now by their topological character in the form of a mapping in an extended Schläfli space, as is shown in Figure 2 below.

\section{Figure 2}

\section{A Survey of the Topology of Structures}

As Equations (1) \& (2), and Figure $1 \& 2$ explicitly reveal, it is the Platonic solids that form the basis of this mapping formulation of structures described in this paper. These forms, as shown in Figure 3 with their appropriate 
polyhedral face symbolism [10], as discovered in Ancient Greece from the application of pure thought, were implicated later on in Plato's Timaeus, as the building blocks of Nature [11]. With the advent of modern crystallographic techniques by the Bragg's in the $20^{\text {th }}$ century [12], we have come to learn that the structure of matter does indeed often take on various vestiges of these eternal objects. And so they have come to be important in modern structural chemistry as elucidated by Pauling [13] and others.

\section{Figure 3}

The polyhedra, thus forming the basis of the topology map of structures in Figure 2, and also rigorously obeying the topology relations shown as Equations (1) \& (2) above, are positioned uniquely in this construction to support the vast space of tessellations and networks that, as we have seen in the preceding Section, can be mapped, rigorously, in Figure 2 by the identification and proper translation of their Well's point symbols, as described above, into ordered pairs as Schläfli symbols (n, p). Plato's great work, Timaeus, thus predicted the ascendancy of the material world into perfect forms, in which the Platonic polyhedra hold primacy and support the overall organizational structure of matter, from which the innumerable other polyhedral objects, and the innumerable 2D tessellations, and the innumerable 3D networks, together all emerge as perfect objects, in this scheme.

Later, it has been shown by Duchowicz et al. [14], that indeed molecular structures can be represented in the scheme of Figure 2, and they have a corresponding set of two topology relations (in addition to $\mathrm{n}=2 \mathrm{E} / \mathrm{F}$ and $\mathrm{p}=2 \mathrm{E} / \mathrm{V}$ ), shown as Equations (4) \& (5) below, that govern their mapping into Figure 2. 


$$
\begin{aligned}
& \mathrm{V}-\mathrm{E}+\mathrm{F}=1 \\
& \frac{1}{n}-\frac{1}{2}+\frac{1}{p}=\frac{1}{2 E}
\end{aligned}
$$

In this way, one can see that the overall chemical topology scheme described here, is a complete description of the topology of all matter constituting the material world. This communication will not treat the specific applications of Equations (4) \& (5), but it will be left to the reader to refer to those applications suggested in the literature [14].

Moving from the polyhedra and molecular fragments, as described above, one can then map the regular tessellations as shown in Figure 4, with the honeycomb tessellation, given by the Wells point symbol notation as $6^{3}$, and translated into the mapping symbol or Schläfli symbol as $(n, p)=(6,3)$, and the square grid, given by the Wells point symbol notation as $4^{4}$, and translated into the mapping symbol or Schläfli symbol as $(n, p)=(4,4)$, and finally, the third regular tessellation, which thus outlines the space of Figure 2 in terms of the tessellations, as the closest-packed grid, given by the Wells point symbol notation as $3^{6}$, and translated into the mapping symbol or Schläfli symbol as $(n, p)=(6,3)$.

\section{Figure 4}

One can, of course, insert all manner of hybrid tessellations, among these 3 regular ones, and generate innumerable Archimedean, Catalan and Wellsean tessellations. Some hybrid tessellations of the square grid-honeycomb grid 
pair have been analyzed topologically by Bucknum et al. [15]. There are, of course, an infinity of such structural tessellations, and they indeed fill the space in the neighborhood of the borderline between the polyhedra and the tessellations, on the one hand, and the tessellations and the networks in 3D, on the other hand. It is also true that Wells and others [4], have identified tessellations of the plane comprised of 5-gons \& 7-gons, and their have been tessellations of 4-gons \& 6-gons that admit unstrained 8-gons, and there are many more tessellations proposed, some of which have been taken as models of various $\mathrm{C}$ allotropes [16-18], which essentially can possess any n-gons in their pattern, provided that the restraint of being regular n-gons is relaxed. And it is thus true, that all of this infinity of tessellations can be mapped, rigorously, by the methods outlined above.

Finally, the map in Figure 2 outlines the 3D networks, and a prominent member is, of course, the diamond lattice given by the Wells point symbol $6^{6}$, which is translated [9] into the Schläfli symbol $(6,4)$. By examination of Figure 2, one can see that the diamond network, given the Schläfli symbol (6, 4), is situated just across the borderline from the 2-dimensional honeycomb tessellation given by $(6,3)$ in the map. One member of the diamond network topology, is in a cubic symmetry space group of Fd-3m, space group \#227, one of the highest symmetry space group patterns. There are, in fact, innumerable possible polytypic patterns within the diamond topology, several of these have been discussed recently by Wen et al. [19] in some detail, and all of them collectively possess the same Wells point symbol of $6^{6}$, and the corresponding Schläfli symbol of $(6,4)$. It is only by their symmetry character, that the members of the diamond polytypic series can be distinguished from each other. Thus, the simplest cubic diamond polytype, known as $3 \mathrm{C}$, is shown in Figure 5 below. 


\section{Figure 5}

Thus in the diamond network, which corresponds to the Platonic (integer) topology of the Platonic polyhedra, one can readily trace the uniform 6-gon, puckered circuitry of the network connected together by all 4-connected, tetrahedral vertices. Diamond's topology classifies the network as a regular, Platonic structure-type.

Next, we move to the space between $(6,3)$ and $(6,4)$, seemingly between $2 \mathrm{D}$ and $3 \mathrm{D}$ forms, and investigate what potential structures might emerge along this boundary area. Such an examination turns up two distinct families of Catalan networks, that together possess the Catalan Wells point symbol $\left(6^{6}\right)_{\mathrm{x}}\left(6^{3}\right)_{\mathrm{y}}$. One can see, through this Wells point symbol notation, that we are describing hybrid structures of the honeycomb tessellation, the socalled graphene grid, $6^{3}$, and the diamond network, $6^{6}$. The notation " $\mathrm{y} / \mathrm{x}$ " specifies the stoichiometry of the net, in terms of the ratio of 3-connected, trigonal planar vertices, to 4-connected, tetrahedral vertices in the hybrid structure [20].

Thus one example of such a class of hybrid "graphene-diamond" structures is shown in Figure 6, and these forms are known, by their hybrid topology, as the "graphite-diamond hybrids". They come in infinite series', in each of two varieties that are known as the ortho- form, and the para-form, these have been elegantly described by Balaban et al. in 1994 [21]. Collectively, as a family, they possess the Schläfli symbol of $\left(6,3^{(\mathrm{x} /(\mathrm{x}+\mathrm{y}))}\right)$, where the parameters " $x$ " and " $y$ " have the stoichiometric significance ascribed to them in the preceding paragraph. These structures, as a family, 
occupy the border-line area between $(6,3)$ and $(6,4)$ in the Schläfli map in Figure 2.

\section{Figure 6}

Yet another family of "graphite-diamond" hybrid structures to be considered, with the Wells point symbol $\left(6^{6}\right)_{x}\left(6^{3}\right)_{y}$, and the corresponding Schläfli symbol given by $\left(6,3^{(x /(x+y))}\right)$, is the family of structures described first by Karfunkel et al. [22] in 1992, as being built from the barrelene hydrocarbon molecular fragment, and extended by the insertion of benzenelike tiles to the parent framework, to generate many infinities of derived structures, which all, collectively, possess the hybrid graphite-diamond topology described above. Later, in 2001, Bucknum [23] clarified the details of the parent such structure derived by Karfunkel et al., and he called this structure "hexagonite" and the derived, such structures were known as the "expanded hexagonites". This name was assigned due to the symmetry space group of the parent structure, in P6/mmm, space group \#191, and also due to the topology of the family of such structures, in which all circuitry over all members of the family, are comprised of 6-gons. The topological analysis of the hexagonite family, suggests that they begin with the Schläfli symbol (n, p) $=\left(6,3^{2 / 5}\right)$, and extend from there, in descending, discrete increments of the connectivity, $p$, towards their termination at $(n, p)=(6,3)$, in the limit of the graphene grid topology. The parent "hexagonite" is shown in two views in Figure 7.

\section{Figure 7}


It should be noted here that the so-called "graphite-diamond hybrids", as described above, are models for novel types of allotropes of carbon. In the vein of this discussion, it should be mentioned here that the border-line space in the topology map of Figure 2, between the entry in the map of $(5,3)$, which is the pentagonal dodecahedron, and the entry in the map $(6,3)$ which is, of course, the graphene grid, lies the collective and infinite space of the fullerene C structures [24]. So, we can see that thus, the collective Schläfli symbol for the family of fullerenes is, in fact, given by $\left(5^{(\mathrm{x} /(\mathrm{x}+\mathrm{y}))}, 3\right)$, where, in this instance, " $x$ " is the number of pentagons in the polyhedron, and " $y$ " is the number of hexagons in the polyhedron [10]. The Schläfli symbol for the parent $\mathrm{C}$ allotrope called "Buckminsterfullerene" $\left(\mathrm{C}_{60}\right)$ is $\left(5^{5 / 8}, 3\right)$ [24], and as substitution into Equation 2 will show, this Schläfli symbol rigorously describes the Buckminsterfullerene polyhedron fully. Figure 8 shows a view of this polyhedron of icosahedral symmetry, and it is clear from this view that the polyhedron is uniformly 3-connected, (as the Schläfli symbol reveals, the fullerenes are Archimedean) and comprised entirely of 5-gon and 6-gon circuitry.

\section{Figure 8}

Yet a final 3D network to be mentioned in this connection, which is a model of a 3-,4-connected network of C, as opposed to a straight "graphitediamond" hybrid, lying between $(6,3)$ and $(6,4)$ of Figure 2 , or a fullerene polyhedron, lying between $(5,3)$ and $(6,3)$ of Figure 2 , as in the preceding discussions with respect to allotropes of $\mathrm{C}$; is the so-called "glitter" network of C invented by Bucknum et al. in 1994 [25]. This structure can be 
envisioned as being constructed from a 1,4-cyclohexadiene building block, and it is shown in Figure 9.

\section{Figure 9}

As can be seen in Figure 9, the Wells point symbol for this Wellsean network is given by $\left(6^{2} 8^{4}\right)\left(6^{2} 8\right)_{2}$, and derived from this, is its Schläfli symbol of ( 7 , $3^{1 / 3}$ ) [9]. It is comprised of 6 -gons admixed with 8 -gons, in its topology, and an admixture of two trigonal vertices for every one tetrahedral vertex in its connection pattern. This particular $\mathrm{C}$ network has been important from the perspective of the 3-dimensional (3D) resonance structures which can be drawn over it, see Figure 10, and there have been some favorable indications that its synthesis has been achieved [26-27].

\section{Figure 10}

Other inorganic networks that are of interest, that are not $\mathrm{C}$ allotropes, or models of $\mathrm{C}$ allotropes, would include the Archimedean Cooperite network, the structure of the minerals PtS and PdO [13], shown in Figure 11, which is a 4-connected network comprised of an equal mixture of tetrahedral and square planar vertices, both of which are distorted in their geometries [22].

\section{Figure 11}

As Figure 11 indicates, taking both tetrahedral and square planar vertices as equally 4-connected, one can thus assign an Archimedean topology to this 
network, with a Wells point symbol of $\left(4^{2} 8^{4}\right)\left(4^{2} 8^{2}\right)$, and a Schläfli symbol of $\left(6^{2 / 5}, 4\right)[9]$. In this case, the network nominally is binary, with two distinct types of connectivity, and an apparently Wellsean topology associated with this, but, in fact in this instance, we have a 4-connected network where the square planar vertices (with 4 independent circuits) are viewed as equivalent in topology (if distorted) versions of the tetrahedral vertices (with 6 independent vertices).

Yet another inorganic network includes, but is not limited to, the Catalan fluorite network [13], the structure of a number of mineral fluorides including $\mathrm{CaF}_{2}$, shown in Figure 12.

\section{Figure 12}

This densely connected network is comprised of 4-connected tetrahedral vertices, and 8-connected cube-centered vertices, which are connected to each other through a uniform set of 4-gons. The topology of this network can be represented by the Wells point symbol $\left(4^{24}\right)\left(4^{6}\right)_{2}$, and this can be translated into a Catalan Schläfli symbol of $\left(4,5^{1 / 3}\right)$ [9]. It can be mapped in Figure 2 just beyond the entry $(4,5)$.

Still another inorganic structure-type we can provide the topology of, is the rocksalt (or primitive cubic) lattice [13], which is the structure of a number of inorganic alkali metal halides and alkaline earth chalcogenides. The rocksalt lattice is shown in Figure 13 below.

Figure 13 
Also known as the primitive cubic structure-type, the rocksalt lattice is comprised uniformly of octahedral 6-connection in all 4-gon circuitry. The Wells point symbol for the network is given as $4^{12}$, and this can be translated into a Schläfli symbol for the network of $(4,6)$ [9]. Because this rocksalt network is of a Platonic (regular, integer) topology, it can readily be seen where it maps in Figure 2. It represents, structurally, an extension into 3dimensions (3D) of the square grid $4^{4}$, or $(4,4)$ which extends in $2 \mathrm{D}$, through a layering, in exact register, of other square grids onto a parent square grid, and their interconnection through perpendicular interlayer bonding through the respective vertices.

As a final inorganic structural-type that we can analyze here topologically in this Section, we have the so-called body-centered cubic (bcc) structure of $\mathrm{CsCl}$ [13], and a number of inorganic structures including alkali metal halides and alkaline earth chalcogenides and other materials. The bcc structure-type is shown here in Figure 14.

\section{Figure 14}

It is uniformly comprised of 8-connected, cube-centered vertices that are mutually interconnected by all 4-gon circuits in the net. The topology of the bcc lattice can be specified by $4^{24}$, or by the corresponding Platonic Schläfli symbol of $(4,8)[9]$.

There are, of course, innumerable other network structures that possess 3-dimensional geometries, one only has to look at the exhaustive works of O'Keeffe et al. to discern the scope of this field [28]. In the present discussion, we move, in the next Section, into the area of the topological analysis of some networks in 3-dimensions that exhibit rather odd Schläfli 
symbols (n, p). These networks, the oldest of which was identified only in 1951 (Waserite) [29], and the others of which were identified only in 1988 (Kentuckia) [30], and 2005 (Moravia) [31], suggest from the computation of their respective Schläfli symbols (n, p), by the methods outlined in Section 2 and Equation (3) above, that such numbers represent not only topological parameters of these networks, but they coincidently and fortuitously can be construed as rational approximations, in various instances, to the transcendental mathematical constants $\phi$, e and $\pi$.

\section{Rational Approximations to $\phi$, e and $\pi$ and the Topology of Matter}

Certain material networks, including the $\mathrm{CaCuO}_{2}$ [30] (Kentuckia network [32]) structure-type, that is the progenitor for all of the superconducting cuprates, and the so-called Moravia structure-type [33], that is the proto-type structure for a number of coordination network (also called metal-organic frameworks or MOF's) structural compositions [31], and the so-called Waserite structure-type [29], that is the structure of the anionic, platinate sublattice of the ionic conducting lattice, known as sodium platinate $\left(\mathrm{NaPt}_{3} \mathrm{O}_{4}\right)$, have here, through topological analysis, been shown, either to have a network polygonality, n, (Kentuckia network) the value of which serves as a rational approximation to the transcendental mathematical constant $\pi$, or, alternatively, they have a network connectivity, $\mathrm{p}$, the value of which serves as a rational approximation to the product of the transcendental mathematical constants e and $\phi$ (Moravia network), or e and $\pi$ (Waserite network).

The Kentuckia structure-type, proposed by Bucknum et al. in 2005 [32], is the pattern adopted by the high temperature superconducting cuprate 
composition, it is $\mathrm{CaCuO}_{2}$ [30] that adopts this pattern, which is the progenitor to all the superconducting cuprates discovered so far. This tetragonal structure-type lies in space group P4/mmm, \#123 and is shown in Figure 15 below.

\section{Figure 15}

It can be seen from Figure 15 that this tetragonal oxide, bears a relation to the cubic perovskite $\left(\mathrm{BaTiO}_{3},[13]\right)$ structure-type which lies in the cubic space group Im-3m, not shown here, in which, by removal of an axial pair of oxygen vertices, one can generate the Kentuckia structure-type from the perovskite structure-type. However, it should be pointed out that the perovskite structure-type is a 6-,12-connected network, in which the transition metal titanium and chalcogenide oxygen centers, attain octahedral 6-coordination, while the alkaline earth barium cation bears a closest packed coordination sphere of 12. Whereas in the Kentuckia structure-type, the lattice, by great contrast as can be seen in Figure 15, bears an oxygen vertex with a square planar, 4-connected coordination, and the transition metal copper vertex bears an octahedral, 6-connected coordination, while the alkaline earth calcium cation is in cube-centered, 8 -fold coordination. It is a ternary, 4-,6-,8-connected tetragonal structural pattern, as is described in Figure 15.

Thus the connectivity in Kentuckia, is that of a ternary 4-,6-,8connected structural-type, while the connectivity in perovskite appears to be that of a binary 6-,12-connected network topology. It appears that all the circuitry in perovskite is comprised of 4-gons with, perhaps, the stoichiometry $\mathrm{AB}_{4}$, in which the vertex " $\mathrm{A}$ " is in 12-connected, closest-packed topology, 
and the vertex " $\mathrm{B}$ " is 6-connected, octahedral coordination. This leads to a Wells point symbol for perovskite of $\left(4^{60}\right)\left(4^{12}\right)_{4}$ and a Schläfli symbol of (n, $\mathrm{p})=\left(4,7^{1 / 5}\right)$. Where, as a reference, the hexagonal closest packed (hcp) and cubic closest packed (ccp) networks, have the Wells point symbol $3^{60}$, and a Schläfli symbol of $(n, p)=(3,12)$. In contrast, we see that the polygonality in the Kentuckia pattern, as revealed in Figure 15, is a composite of 4-gons and 6-gons, with the Wells point symbol for the lattice being $\left(4^{4}\right)\left(4^{12} 6^{12}\right)\left(4^{12}\right)_{2}$. As Equation (6) reveals, the polygonality, n, for this structural-type, bears an odd resemblance to the transcendental mathematical constant $\pi$ [31], occurring as it does, within $1 \%$ of exactly the value of $\sqrt{ } 2 \cdot \pi$.

$$
\begin{gathered}
\mathrm{n}=(40 \cdot 4+12 \cdot 6) / 52 \\
\mathrm{n}=\sqrt{ } 2 \cdot \pi
\end{gathered}
$$

Turning to the Waserite network [29], which is shown to be a relatively simple, binary 3-,4-connected network topology in Figure 16.

\section{Figure 16}

As is revealed in Figure 16, the 3-to-4 stoichiometry of 4-connected square planar vertices to 3-connected trigonal planar vertices, present in the Waserite topology, together with the Wells point symbol for this network as $\left(8^{4}\right)_{3}\left(8^{3}\right)_{4}$, thus demonstrates that this simple structure is indeed a binary, Catalan network comprised of all 8-gon circuitry. Therefore it is apparent, readily, that the polygonality is simply given by $\mathrm{n}=8$, in this pattern. But as has been 
described previously for this so-called Waserite network [34], the connectivity index of it, as shown in Equations (7), suggests that its topology is more complex than meets the eye.

$$
\begin{gathered}
\mathrm{p}=(3 \cdot 4+4 \cdot 3) / 7 \\
\mathrm{p}=(2 / 5) \mathrm{e} \cdot \pi
\end{gathered}
$$

As Equation 7 reveals, it is a fact of simple arithmetic that the weighted average connectivity of the Waserite network, given by the symbol $\mathrm{p}$, is in fact equal, to better than 99 parts in 100 , to $(2 / 5) \mathrm{e} \cdot \pi$. Here $\pi$ is, the familiar ratio of a circle's circumference to its diameter [35], and e is the natural base of exponentials [36]. These numbers are transcendental, as they are infinite, non-repeating fractions $[35,36]$. An identical relation will also hold for the other structures patterned on a stoichiometry of four 3-connected vertices-tothree 4-connected vertices including, for example, the rhombic dodecahedron (given by the Catalan Wells point symbol as $\left.\left(4^{4}\right)_{6}\left(4^{3}\right)_{8}\right)$, and the well-known phenacite network of Bragg et al. (not shown, given by the Wellsean ternary Wells point symbol $\left.\left(8^{3}\right)\left(6^{3}\right)_{3}\left(6^{3} 8^{3}\right)_{3}[37]\right)$

Other relations emerging from such consideration of the connectivity index, p, in the Waserite structural-type include Equation (8) [34], known hereafter as the Timaeus relation, for its suggestion of a Cosmogony based upon the 5 Platonic solids as enunciated by Plato [11].

$$
(1) \cdot(2.3333333 \ldots \ldots \ldots \ldots) \cdot e \cdot \pi=(4) \cdot(5)
$$


Equation (8) suggests the ultimate simplicity of definitions of e and $\pi$, through an elementary relationship involving only the first 5 counting numbers, or alternatively, the first 4 prime numbers [34].

Finally, in this survey of crystalline structure-types which exhibit relations to the transcendental mathematical constants, in their structural topology, we turn to the so-called Moravia network [31, 33], first posited as a potential structural-type in 2005 by Bucknum et al. This Moravia network has, in fact, turned out to be the structure adopted by several coordination networks known as metal-organic-frameworks (MOF's) [31]. It is readily seen to be a Wellsean, 3-,8-connected network upon careful inspection of the drawing for valences, and tracing of circuitry in Figure 17.

\section{Figure 17}

The Wells point symbol for the Moravia structural-type is encoded as $\left(4^{4} 6^{8} 8^{12}\right)_{3}\left(4^{3}\right)_{8}$, it is thus a complex, Wellsean network composed of two connection motifs, the trigonal planar, 3-connected, and cube-centered, 8connected, vertices, held together in circuits of 4-gon, 6-gon and 8-gon sizes. The complex Well's point symbol for the network, belies in this instance, the relatively high symmetry of the structure, in which Moravia is lying in the cubic space group Pm-3m, \#221.

If we take the point symbol for the network, and analyze it according to Equations (3) described above, the Well's point symbol translation formulas, one obtains the result that the weighted average polygonality for the network is indeed given by $n=6$. The Waserite net is thus pseudo-Catalan, with an integer polygonality of 6 , that is nonetheless the result of averaging over 4-, 6- and 8-gons in its structural pattern. Upon calculating the 
connectivity index, $\mathrm{p}$, for Moravia, however, we get the result shown in Equation (9).

$$
\begin{aligned}
& \mathrm{p}=(3 \cdot 8+8 \cdot 3) / 11 \\
& \mathrm{p}=\mathrm{e} \cdot \phi
\end{aligned}
$$

Thus it is seen that the connectivity index, p, of Moravia, as a 3-,8-connected network, is equivalent, to better than 99 parts in 100 , to the product of the two transcendental mathematical constants $\phi[38]$ and e [36], given simply as $\phi \cdot e$. Here, as above, e is the natural base of exponentials [36], and $\phi$ is the well-known golden ratio [38], as is expressed in Equation (10).

$$
\phi=(\sqrt{5}+1) / 2
$$

Equations (9), like the transformation of Equation (7) to Equation (8) above, can be factored, interestingly, so that the relation of $\phi \cdot e$ evolving out of the topology of the Moravia structure, as shown in Figure 17 above, involves the first 6 Fibonacci numbers, F(1-to-6) (given, on the left, in Equation (11b) as $1,1,2,3,5$, and 8$)$, and these are related to the $10^{\text {th }}$ Fibonacci number, $F(10)$ (given, on the right, in Equation (11b) as 55).

$$
\begin{aligned}
& F(1) \cdot F(2) \cdot F(3) \cdot F(4) \cdot F(5) \cdot F(6)=(e) \cdot(\phi) \cdot F(10) \\
& (1) \cdot(1) \cdot(2) \cdot(3) \cdot(5) \cdot(8)=(e) \cdot(\phi) \cdot(55)
\end{aligned}
$$


These relations between the topology of these structures in this Section, as is revealed by the computation and mapping of their corresponding Schläfli symbols ( $n, p)$, and the transcendental mathematical constants $\phi$, e and $\pi$, that can be thus correlated to their structural character, suggest the mathematical, and potentially scientific, richness that such structures may lead to.

\section{Conclusions}

In this paper we have reviewed the basic tenets of a chemical topology scheme, one that can be applied to classify and effectively map the innumerable polyhedra, tessellations and networks, based upon a simple computation of their Schläfli symbols $(n, p)$, from translation of their corresponding Wells point symbols. A restriction pointed up by this work, is that all structures in such a chemical topology scheme must, indeed, be simply connected. The phrase "simply connected" means that all edges, E, in a structure, be it a polyhedron, tessellation or network, must terminate at distinct vertices, $\mathrm{V}$, in the network, where such edges, $\mathrm{E}$, are known as proper edges. A second condition on a net being "simply connected", is that all faces, F, in the structure should be bounded by proper edges, E, as defined in the preceding sentence. It is not clear, at this juncture, what form a systematic chemical topology would take on for the "non-simply connected" structures. As there are innumerable non-simply connected structures, to accompany the infinite number of simply connected structures in Schläfli space (the space of the Schläfli symbols $(n, p)$ ), it would seem that a topological analysis of these complex structures would be desirable and necessary to get a more complete handling of the chemical topology of crystal chemistry. 
The brunt of this paper has been dedicated to a survey of some of the more prominent (well-known or obvious) organic and inorganic structuretypes. Organic structures included some well-known $\mathrm{C}$ allotropes, like the regular, graphene grid and the regular, diamond network, both forms of $\mathrm{C}$ known since Antiquity. And, also, more modern $\mathrm{C}$ forms were surveyed, like the 3-,4-connected, Catalan graphite-diamond hybrids [21], the 3-,4connected, Catalan hexagonite lattices [22, 23] and the Wellsean, 3-,4connected glitter $\mathrm{C}$ form [25], for which there is currently some evidences of their syntheses from the growth of $\mathrm{C}$ nanocrystals [26, 27]. Inorganic structures included in this survey, were the 4-,8-connected, Catalan fluorite lattice, the 4-connected, Archimedean Cooperite lattice, the 8-connected, regular $\mathrm{CsCl}$, body-centered cubic structure-type, and the 6-connected, regular rocksalt structure-type. [13] Finally, in this survey, lattices which admitted connections in their topology to the transcendental numbers included the 3-,8-connected, Wellsean Moravia net, discovered in 2005 [31, 33] (related to $\phi$ and e, through the connectivity), the 4-,6-,8-connected, Wellsean Kentuckia (cuprate structure-type) net, discovered in 1988 [30, 32] (related to $\pi$, through the polygonality), and finally the 3-,4-connected, Catalan Waserite net (platinate structure-type), discovered in 1951 [29].

The occurrence of relations to the transcendental numbers of mathematics, in the computations of the topology character of some of these networks, is indeed a mysterious outcome. It is not clear whether such relations could imply that the topology of these lattices, like the Kentuckia lattice, in which $n=\sqrt{ } 2 \cdot \pi$, could indeed be equated to some type of ordering parameter for the lattice, such that by the introduction of systematic defects in the connectivity, $\mathrm{p}$ (or thereby the polygonality, $\mathrm{n}$ ) over the bulk lattice, might 
lead to a corrected value of $n$, that asymptotically approaches the true value of $\pi$, and that, that might have some bearing on bulk properties of the Kentuckia network, like the critical superconducting transition temperature, $\mathrm{T}_{\mathrm{c}}$, in the cuprate composition $\mathrm{CaCuO}_{2}$ [32]. Such considerations as these, open up new avenues of explorations for solid state scientists based upon the intrinsic topology character of such networks as these. 


\section{References}

[1] M.J. Bucknum, Carbon, 35(1), 1, (1997).

[2] Leonhard Euler, Elementa doctrinae solidorum et Demonstratio nonnularum insignium proprietatum quibus solida heddris planis inclusa sunt praedita, Proceedings of the St. Petersburg Academy, St. Petersburg, Russia 1758 .

[3] J.J. Burckhardt, Der mathematische Nachlass von Ludwig Schläfli, 18141895, in der Schweizerischen Landesbibliothek, $1^{\text {st }}$ edition, Bern, 1942.

[4] A.F. Wells, Three Dimensional Nets and Polyhedra, $1^{\text {st }}$ Edition, John Wiley and Sons Inc., New York, NY 1977.

[5] M. Gardner, Archimedes: Mathematician and Inventor, $1^{\text {st }}$ edition, Macmillan Publishing, New York, NY, 1965.

[6] P.J. Frederico, Descartes on Polyhedra: A Study of the De Solidorum Elementis, $1^{\text {st }}$ edition, Springer-Verlag, New York, NY, 1982.

[7] A.F. Wells, Further Studies of Three-dimensional Nets, American Crystallographic Association (A.C.A), Monograph \#8, $1^{\text {st }}$ Edition, A.C.A Press, Pittsburgh, PA 1979.

[8] I. Peters, Science News, 160(25/26), 396, (2001).

[9] M.J. Bucknum and E.A. Castro, (MATCH) Commun. Math. Comp. Chem., 54, 89, (2005).

[10] It appears that the 5 Platonic polyhedra obey Equation (2) of the text, if one specifies their topology by a Well's point symbol, given by $\mathrm{A}^{\mathrm{a}}$, in which $\mathrm{n}=\mathrm{A}$ and $\mathrm{p}=\mathrm{a}$, and there is no relation between this polyhedral face symbol, $\mathrm{A}^{\mathrm{a}}$, and the computation of $\mathrm{V}, \mathrm{E}$ and $\mathrm{F}$ in the Euler model of Equation (1). It is also the case, that the 5 Platonic polyhedra obey Equation (2) of the text, if one specifies their topology by a polyhedral face symbol, given as $A^{b}$, in 
which $(\mathrm{b} \cdot \mathrm{A})=2 \mathrm{E}, \mathrm{b}=\mathrm{F}$, and $\mathrm{V}$ is identified through inspection of the polyhedron, then $\mathrm{n}=2 \mathrm{E} / \mathrm{F}$ and $\mathrm{p}=2 \mathrm{E} / \mathrm{V}$, by definition.

For the Archimedean polyhedra it appears that it is only possible to specify (n, p), for insertion into Equation (2), by encoding a polyhedral face symbol, given as $\mathrm{A}^{\mathrm{a}} \mathrm{B}^{\mathrm{b}} \mathrm{C}^{\mathrm{c}} \ldots \ldots$, where a is the number of $\mathrm{A}$-gons, in a ratio with $\mathrm{b}$ B-gons etc., for the polyhedron, and thus, in which $(\mathrm{a} \cdot \mathrm{A}+\mathrm{b} \cdot \mathrm{B}+\mathrm{c} \cdot \mathrm{C}$ $+\ldots \ldots.)=2 \mathrm{E},(\mathrm{a}+\mathrm{b}+\mathrm{c}+\ldots \ldots)=\mathrm{F}$, and $\mathrm{V}$ is identified through inspection of the polyhedron, and where finally then, $\mathrm{n}=2 \mathrm{E} / \mathrm{F}$ and $\mathrm{p}=2 \mathrm{E} / \mathrm{V}$, by definition. While for the Catalan and Wellsean polyhedra, by contrast, it appears that it is only possible to specify (n, p), for insertion into Equation (2), by encoding a Wells point symbol, given as $\left(\mathrm{A}^{\mathrm{a}}\right)_{\mathrm{x}}\left(\mathrm{A}^{\mathrm{b}}\right)_{\mathrm{y}}\left(\mathrm{A}^{\mathrm{c}}\right)_{\mathrm{z}} \ldots \ldots \ldots$, where $\mathrm{a}$ is the number of A-gons meeting at $\mathrm{a}$, and $\mathrm{b}$ is the number of A-gons meeting at $\mathrm{b}$ etc., and $\mathrm{x}, \mathrm{y}, \mathrm{z}$ etc. specify the stoichiometry of the polyhedron, for the Catalan or Wellsean polyhedron of interest, in which $(\mathrm{a} \cdot \mathrm{A}+\mathrm{b} \cdot \mathrm{A}+\mathrm{c}$. $A+\ldots \ldots ..)=E,(a \cdot x+b \cdot y+c \cdot z+\ldots \ldots .)=.4 F$ and $(x+y+z+\ldots \ldots .)=$. $\mathrm{V}$, where finally then, $\mathrm{n}=2 \mathrm{E} / \mathrm{F}$ and $\mathrm{p}=2 \mathrm{E} / \mathrm{V}$ by definition, as throughout.

[11] J.M. Cooper, editor, Plato: Complete Works, $1^{\text {st }}$ edition, Hackett Publishing Company, Indianapolis, IN, 1997.

[12] W.L. Bragg, The Development of X-ray Analysis, $1^{\text {st }}$ edition, Dover Publications, Inc., Mineola, NY, 1975.

[13] L. Pauling, The Nature of the Chemical Bond, $3^{\text {rd }}$ edition, Cornell University Press, Ithaca, NY, 1960.

[14] P. Duchowicz, M.J. Bucknum and E.A. Castro, Journal of Mathematical Chemistry, 41(2), 193, (2007). 
[15] (a) M.J. Bucknum and E.A. Castro, (MATCH) Commun. Math. Comp. Chem., 55, 57, (2006). (b) M.J. Bucknum and E.A. Castro, Solid State Sciences, in press, (2008).

[16] R.H. Baughman, H. Eckhardt and M. Kertesz, Journal of Chemical Physics, 87(11), 6687, (1987).

[17] (a) A.T. Balaban, C.C. Rentea and E. Ciupitu, Rev. Roum. Chim., 13, 231, (1968), (b) H. Zhu, A.T. Balaban, D.J. Klein and T.P. Zivkovic, Journal of Chemical Physics, 101, 5281, (1994).

[18] V.H. Crespi, L.X. Benedict, M.L. Cohen and S.G. Louie, Physical Review B, 53, R13303, (1996).

[19] B. Wen, J. Zhao, D.Si, M.J. Bucknum and T.Li, Diamond \& Related Materials, in press, (2008).

[20] M.J. Bucknum and E.A. Castro, Journal of Chemical Theory \& Computation, 2(3), 775, (2006).

[21] A.T. Balaban, D.J. Klein and C.A. Folden, Chemical Physics Letters, 217, 266, (1994).

[22] H.R. Karfunkel and T. Dressler, Journal of the American Chemical Society, 114, 2285, (1992).

[23] M.J. Bucknum, Chemistry Preprint Archive, 2001(1), 75, (2001)

[24] J. Baggott, Perfect Symmetry: The Accidental Discovery of Buckminsterfullerene, $1^{\text {st }}$ edition, Oxford University Press, Oxford, U.K., 1996.

[25] M.J. Bucknum and R. Hoffmann, Journal of the American Chemical Society, 116, 11456, (1994).

[26] M.J. Bucknum and E.A. Castro, Journal of Theoretical \& Computational Chemistry, 5(2), 175, (2006). 
[27] M.J. Bucknum and E.A. Castro, Molecular Physics, 103(20), 2707, (2005).

[28] M. O'Keeffe and B.G. Hyde, Crystal Structures I. Patterns and Symmetry, $1^{\text {st }}$ edition, Mineralogical Society of America (M.S.A.), Washington, D.C., 1996.

[29] J. Waser and E.D. McClanahan, Journal of Chemical Physics, 19, 413, (1951).

[30] T. Siegrist, S.M. Zahurak, D.W. Murphy, R.S. Roth, Nature, 334, 231, (1988).

[31] M. Dincã, A. Dailly, Y.Liu, C.M. Brown, D.A. Neumann and J.R. Long, Journal of the American Chemical Society, 128, 16876, (2006).

[32] M.J. Bucknum and E.A. Castro, Russian Journal of General Chemistry, 76(2), 265, (2006).

[33] M.J. Bucknum and E.A. Castro, Central European Journal of Chemistry (CEJC), 3(1), 169, (2005).

[34] M.J. Bucknum and E.A.Castro, Journal of Mathematical Chemistry, 42(3), 373, (2007).

[35] (a) P. Beckmann, A History of $\pi, 1^{\text {st }}$ edition, The Golem Press, New York, NY, 1971. (b) D. Blatner, The Joy of $\pi, 1^{\text {st }}$ edition, Walker Publishing Company, Inc., USA, 1997.

[36] E. Maor, e: The Story of a Number, $1^{\text {st }}$ edition, Princeton University Press, Princeton, NJ, 1994.

[37] M.J. Bucknum, B. Wen and E.A. Castro, Global Journal of Molecular Sciences, in press, (2008).

[38] M. Livio, The Golden Ratio: The Story of $\phi$, the World's most Astonishing Number, $1^{\text {st }}$ edition, New York, NY, Broadway Books, 2002. 


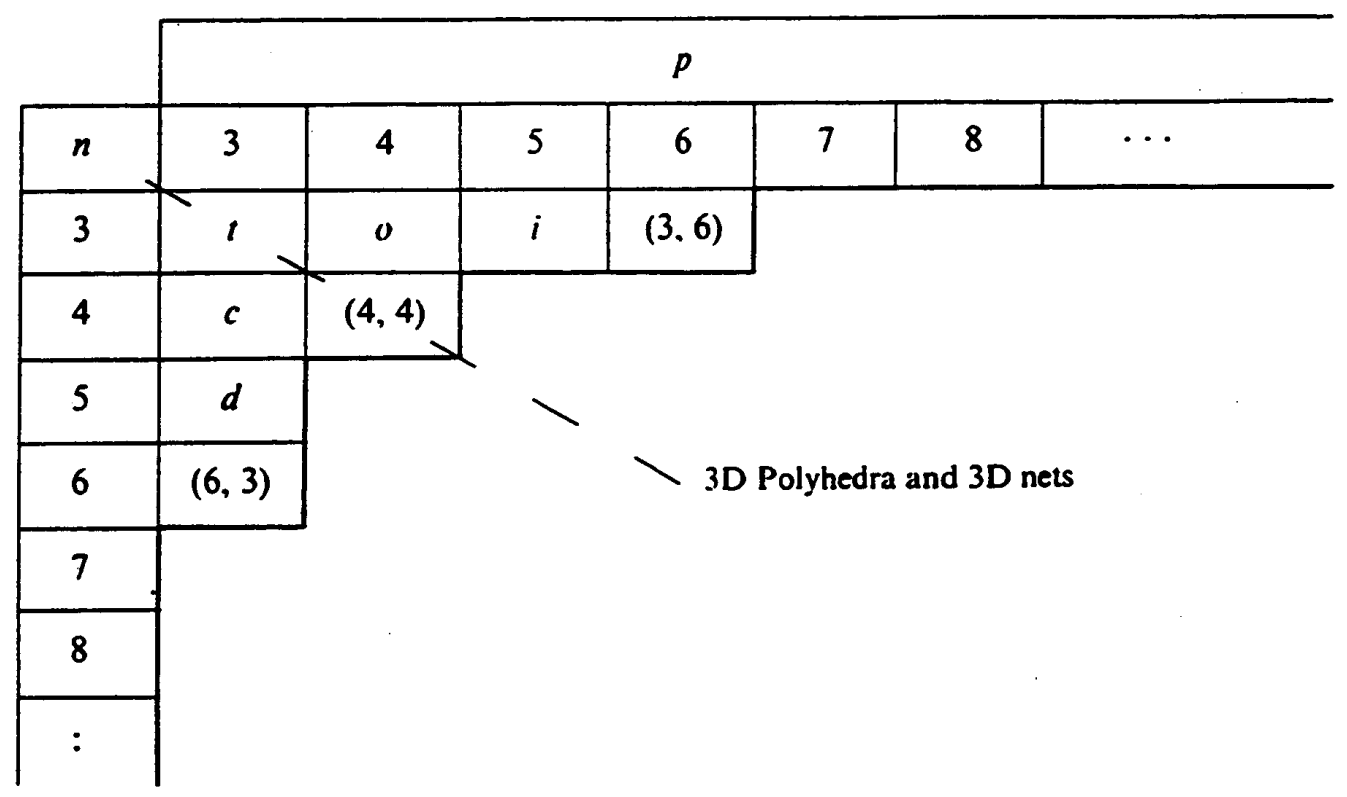

Figure 1: Topology mapping of the Platonic polyhedra due to Wells 
Table 1: Vertex Connectivity, $p$, as a Function of Circuit Number

\begin{tabular}{ccc} 
name & vertex connectivity & circuit number \\
\hline trigonal planar & 3 & 3 \\
square planar & 4 & 4 \\
tetrahedral & 4 & 6 \\
trigonal bipyramidal & 5 & 9 \\
square pyramidal & 5 & 10 \\
octahedral & 6 & 12 \\
cube centered & 8 & 24 \\
anti-cube centered & 8 & 28 \\
closest packed & 12 & 60
\end{tabular}




\begin{tabular}{|c|c|c|c|c|c|c|c}
\hline$n$ & 3 & 4 & 5 & 6 & 7 & 8 & $\ldots$ \\
\hline 3 & 1 & 0 & $\mathrm{i}$ & $(3,6)$ & $(3,7)$ & $(3,8)$ & \\
\hline 4 & $c$ & $(4,4)$ & $(4,5)$ & $(4,6)$ & $(4,7)$ & $(4,8)$ & \\
\hline 5 & $d$ & $(5,4)$ & $(5,5)$ & $(5,6)$ & $(5,7)$ & $(5,8)$ & \\
\hline 6 & $(6,3)$ & $(6,4)$ & $(6,5)$ & $(6,6)$ & $(6,7)$ & $(6,8)$ & \\
\hline 7 & $(7,3)$ & $(7,4)$ & $(7,5)$ & $(7,6)$ & $(7,7)$ & $(7,8)$ & \\
\hline 8 & $(8,3)$ & $(8,4)$ & $(8,5)$ & $(8,6)$ & $(8,7)$ & $(8,8)$ & \\
\hline$\vdots$ & & & & & & & \\
\hline
\end{tabular}

Figure 2: Extended Schläfli space of the Platonic Structures 


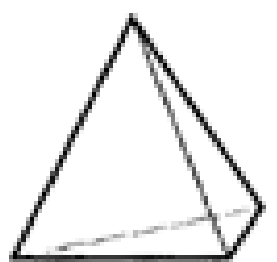

Tetrahedron

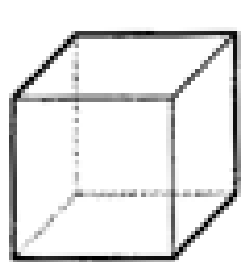

Cube

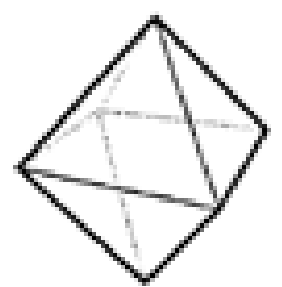

Octahedron

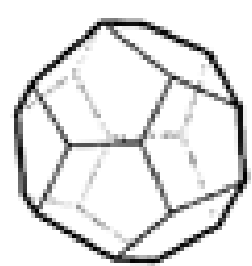

Dodecahedron

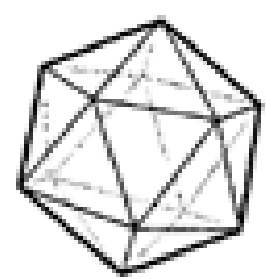

Icosahedron

Figure 3: The Platonic polyhedra, with their corresponding polyhedral face symbols and Wells point symbols, comprised of the tetrahedron $\left(3^{4}\right.$ and $\left.3^{3}\right)$, the octahedron $\left(3^{8}\right.$ and $\left.3^{4}\right)$, the icosahedron $\left(3^{20}\right.$ and $\left.3^{5}\right)$, the cube $\left(4^{6}\right.$ and $\left.4^{3}\right)$, and the dodecahedron $\left(5^{12}\right.$ and $\left.5^{3}\right)$ 


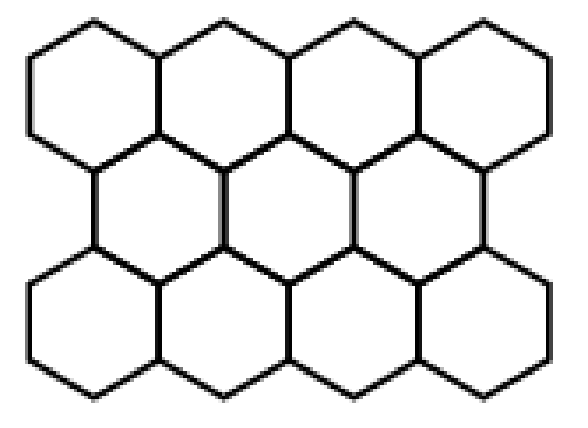

$\{6,3\}$

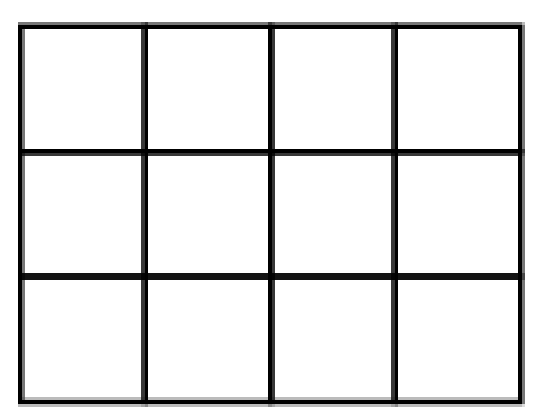

$\{4,4\}$

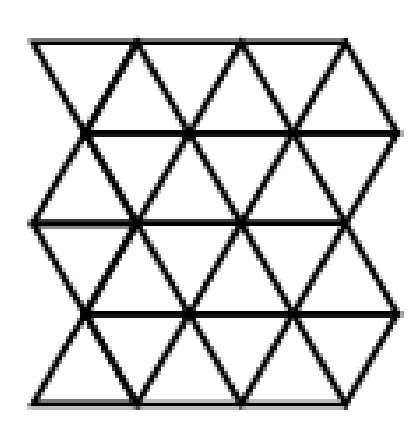

$\{3,6\}$

Figure 4: The Platonic tessellations, with their corresponding Wells point symbols, given as the closest-packed grid $\left(3^{6}\right)$, the square grid $\left(4^{4}\right)$ and the honeycomb grid $\left(6^{3}\right)$ 


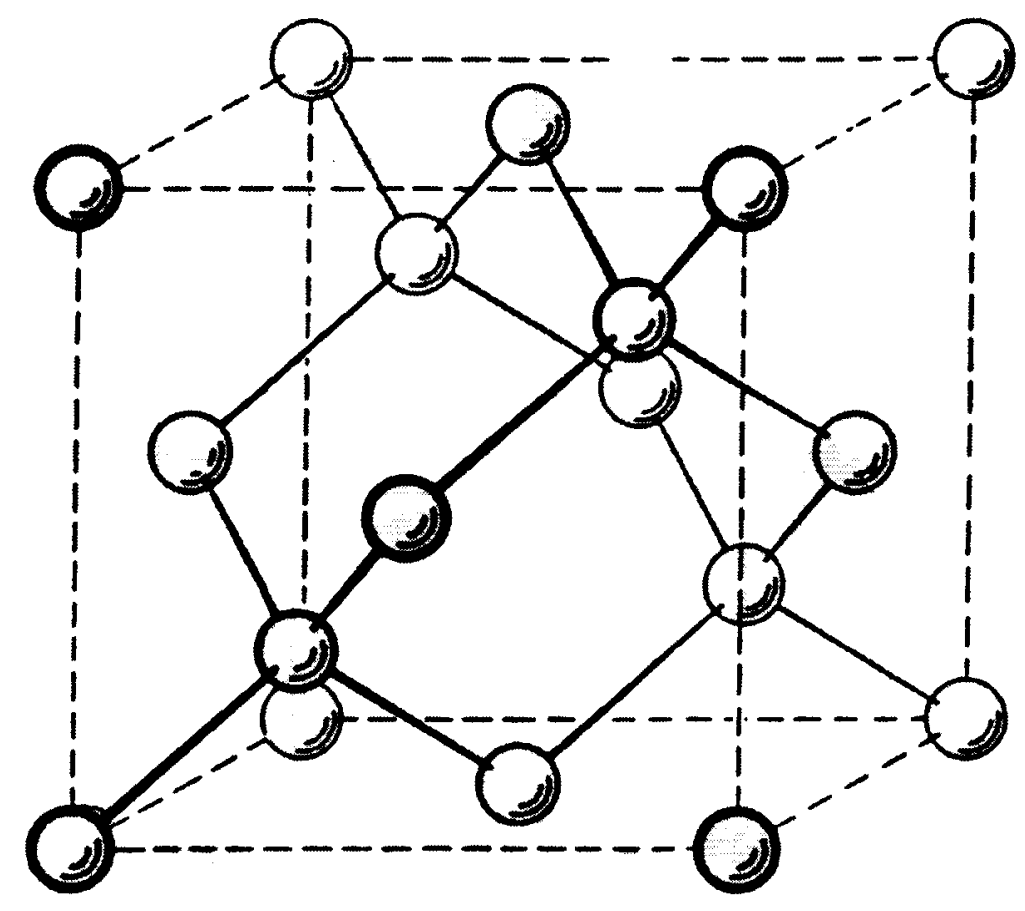

Figure 5: Cubic diamond (3C) polytype, with the Wells point symbol $\left(6^{6}\right)$, lying in symmetry space group (Fd-3m) 

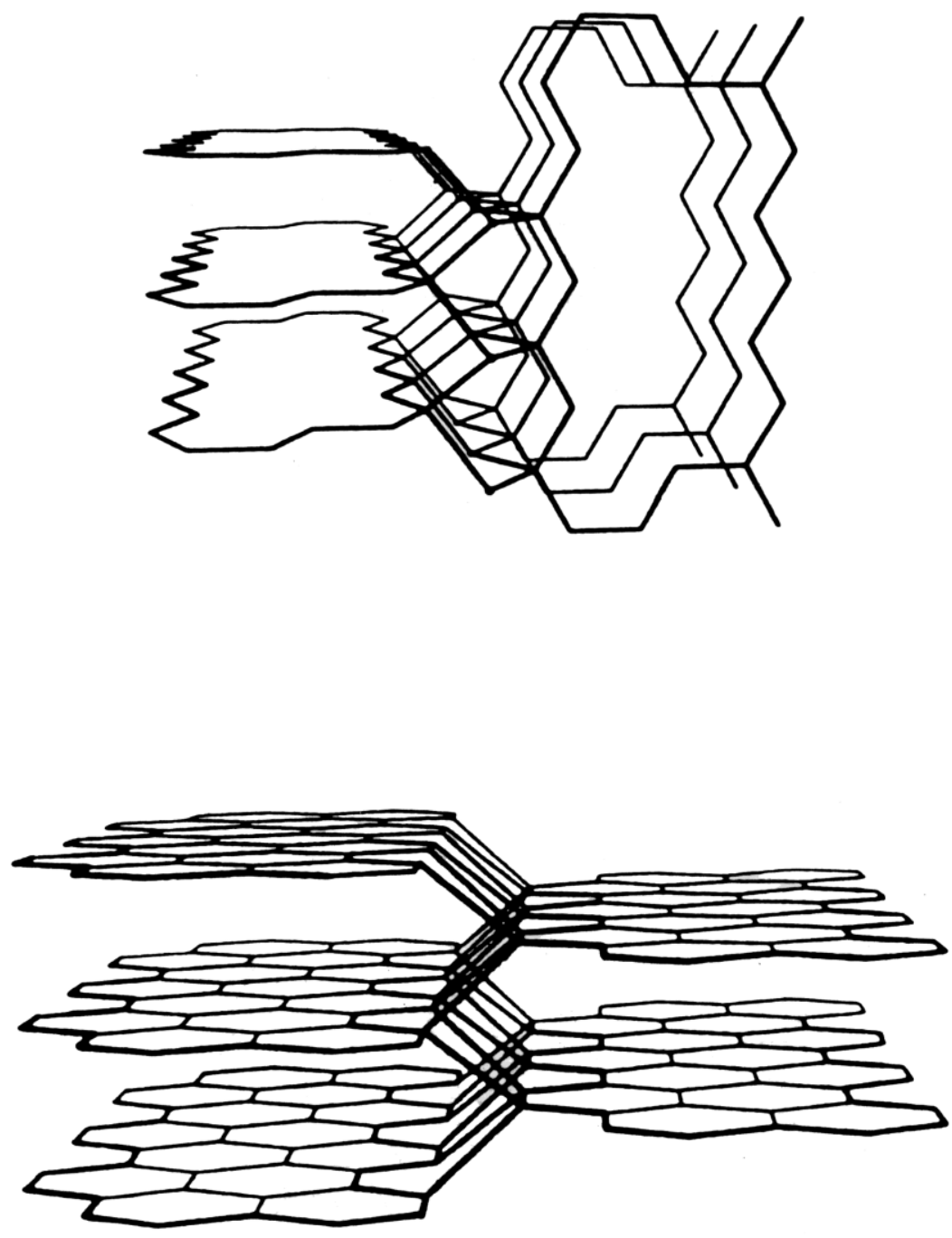

Figure 6: Representatives of the infinite families of ortho- and para- graphitediamond hybrid structures, with the collective Wells point symbol $\left(6^{6}\right)_{\mathrm{x}}\left(6^{3}\right)_{\mathrm{y}}$, of orthorhombic symmetry (Pmmm) 

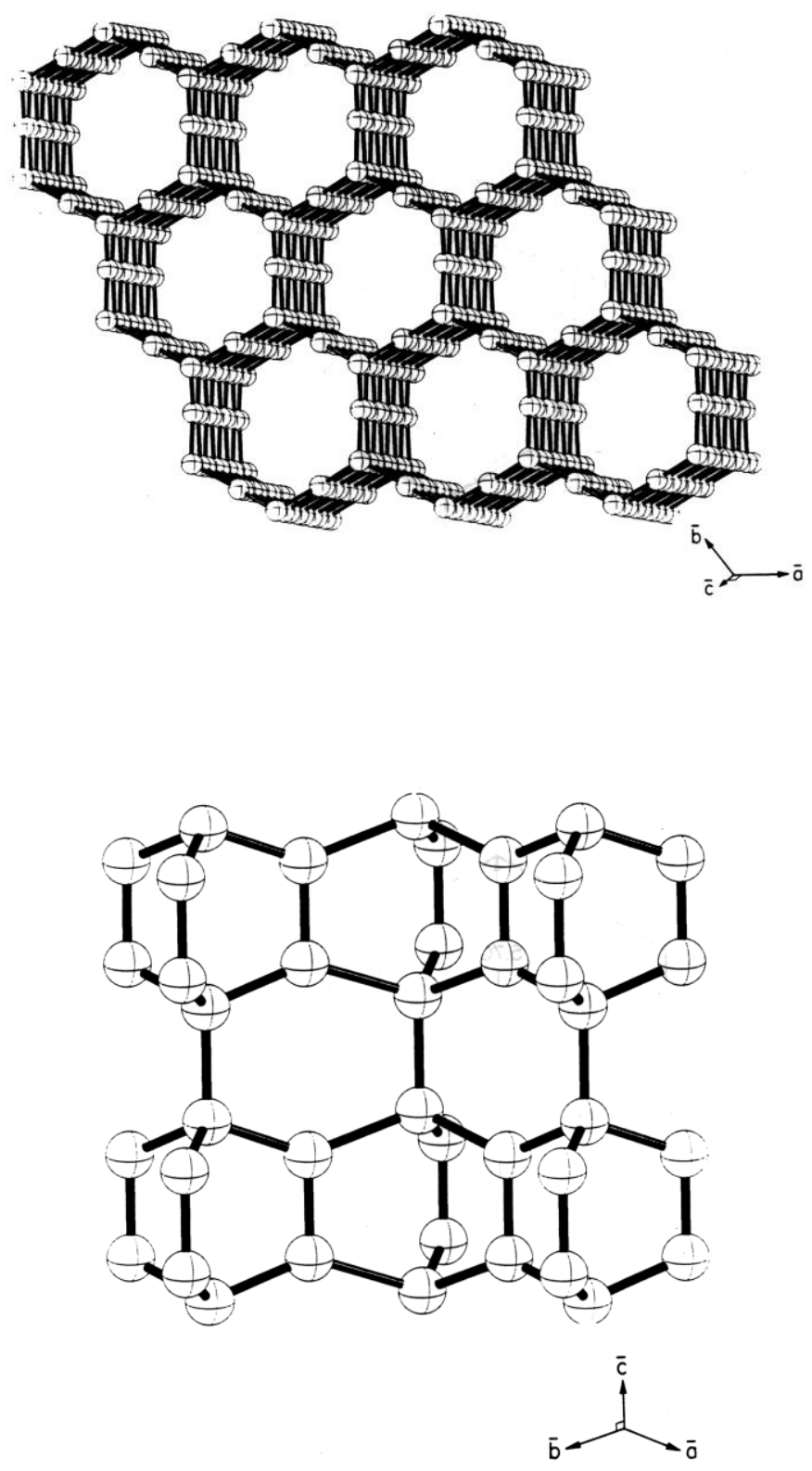

Figure 7: Vertical and lateral views of parent hexagonite structure of the infinite family of hexgonites, with the collective Wells point symbol $\left(6^{6}\right)_{x}\left(6^{3}\right)_{y}$, of orthorhombic-trigonal-hexagonal (Pmmm, P3m1 and P6/mmm) symmetries 


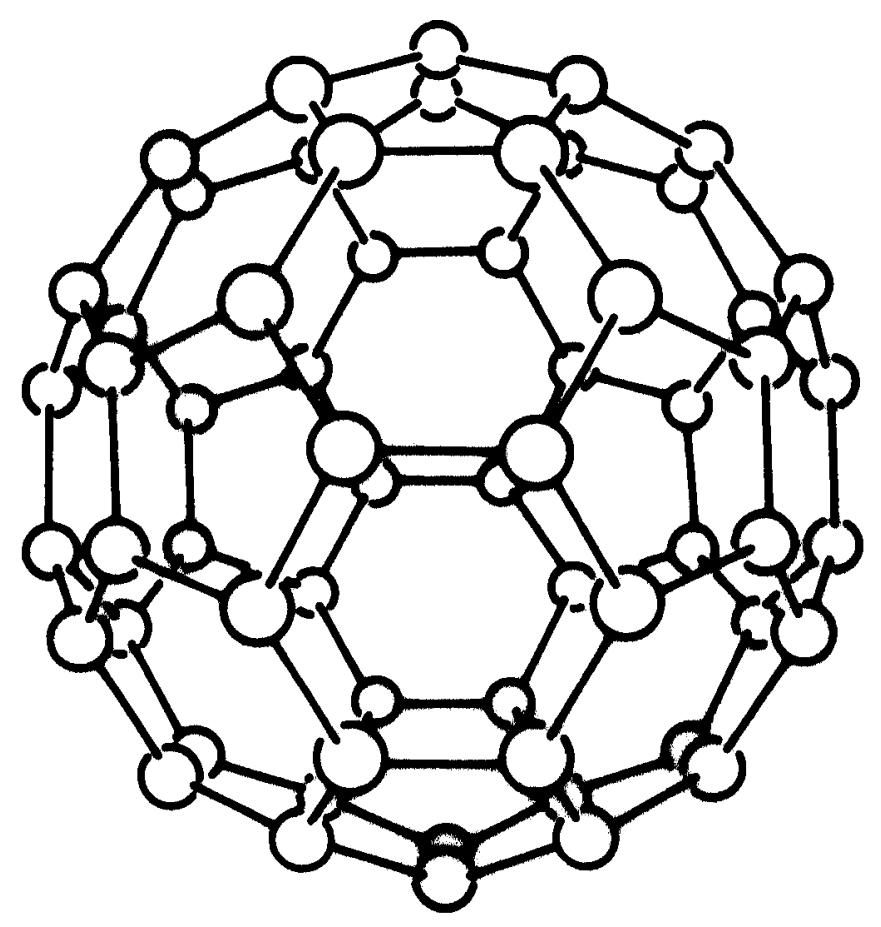

Figure 8: The parent Buckminsterfullerene polyhedron, of the infinite family of fullerenes, with the collective Schläfli symbol for the family of fullerenes given by $\left(5^{(\mathrm{x} /(\mathrm{x}+\mathrm{y}))}, 3\right)$, and the fullerene polyhedral face symbol of $5^{\mathrm{x}} 6^{\mathrm{y}}$, where " $x$ " is the number of hexagons, and " $y$ " is the number of pentagons, in the fullerene, where such structures are of icosahedral $\left(I_{h}\right)$ and lower symmetry 


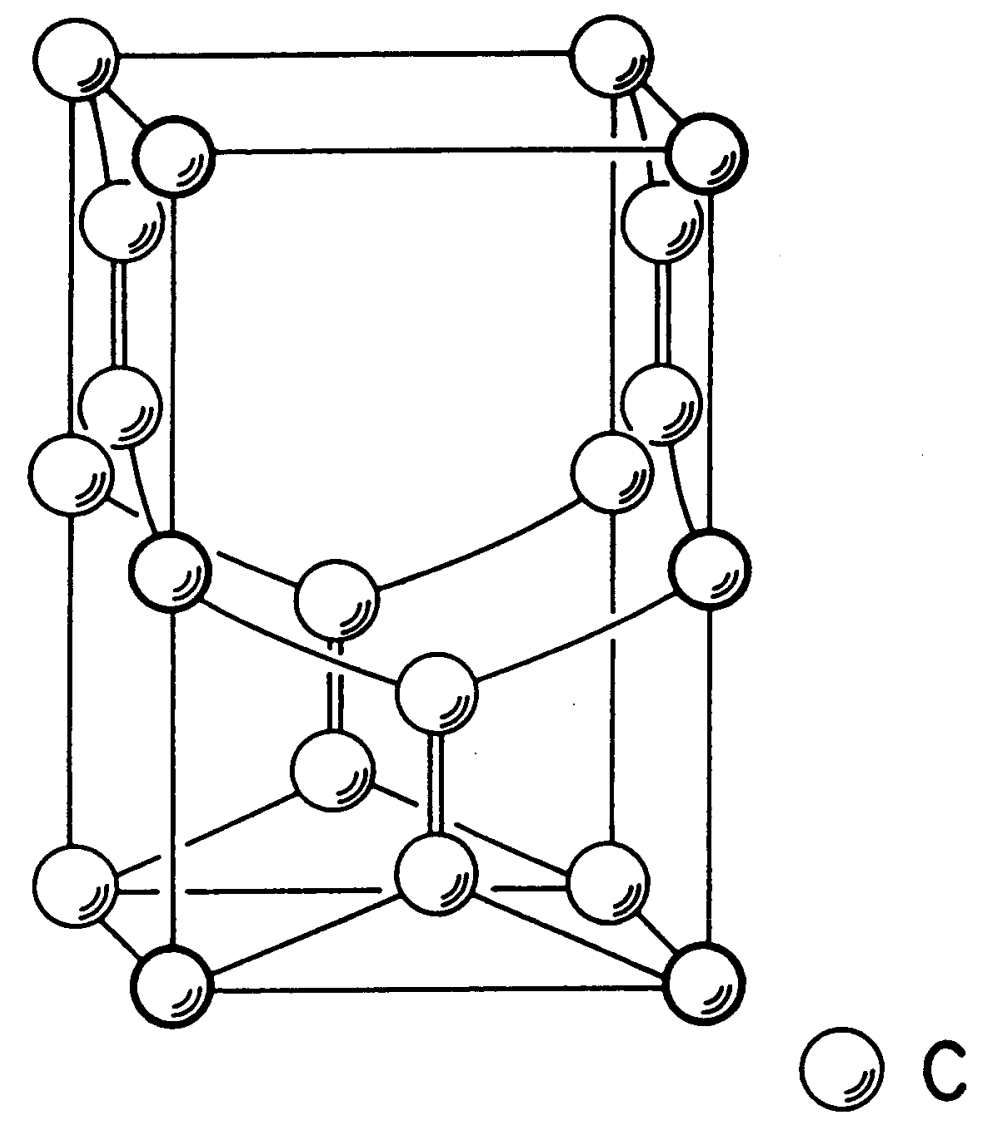

Figure 9: Tetragonal glitter network of carbon, with Wells point symbol given by $\left(6^{2} 8^{4}\right)\left(6^{2} 8\right)_{2}$, and of space group symmetry $\left(\mathrm{P}_{2} / \mathrm{mmc}\right)$ 

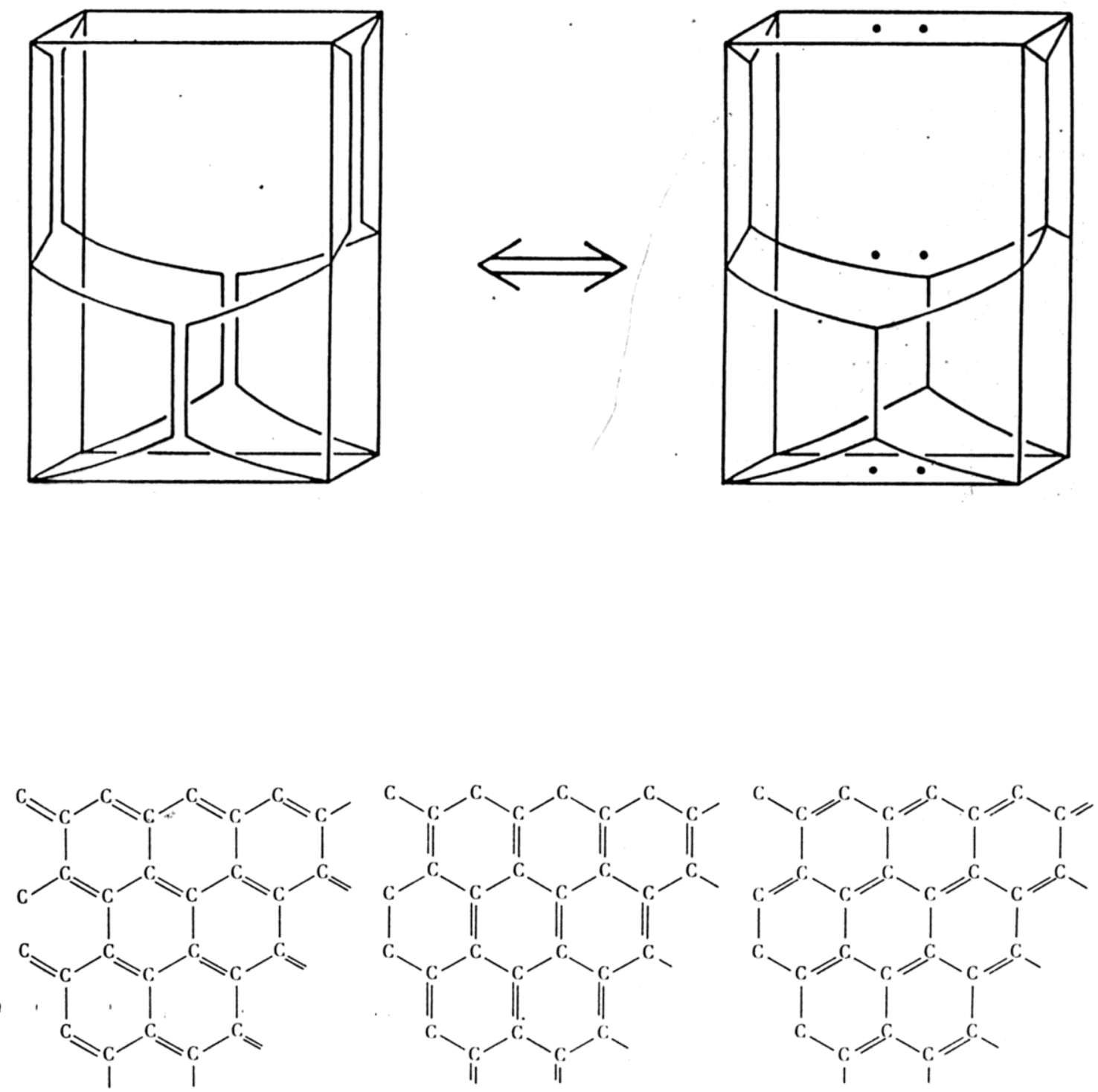

Figure 10: Resonance structures of the graphite and glitter networks of $\mathrm{C}$ 

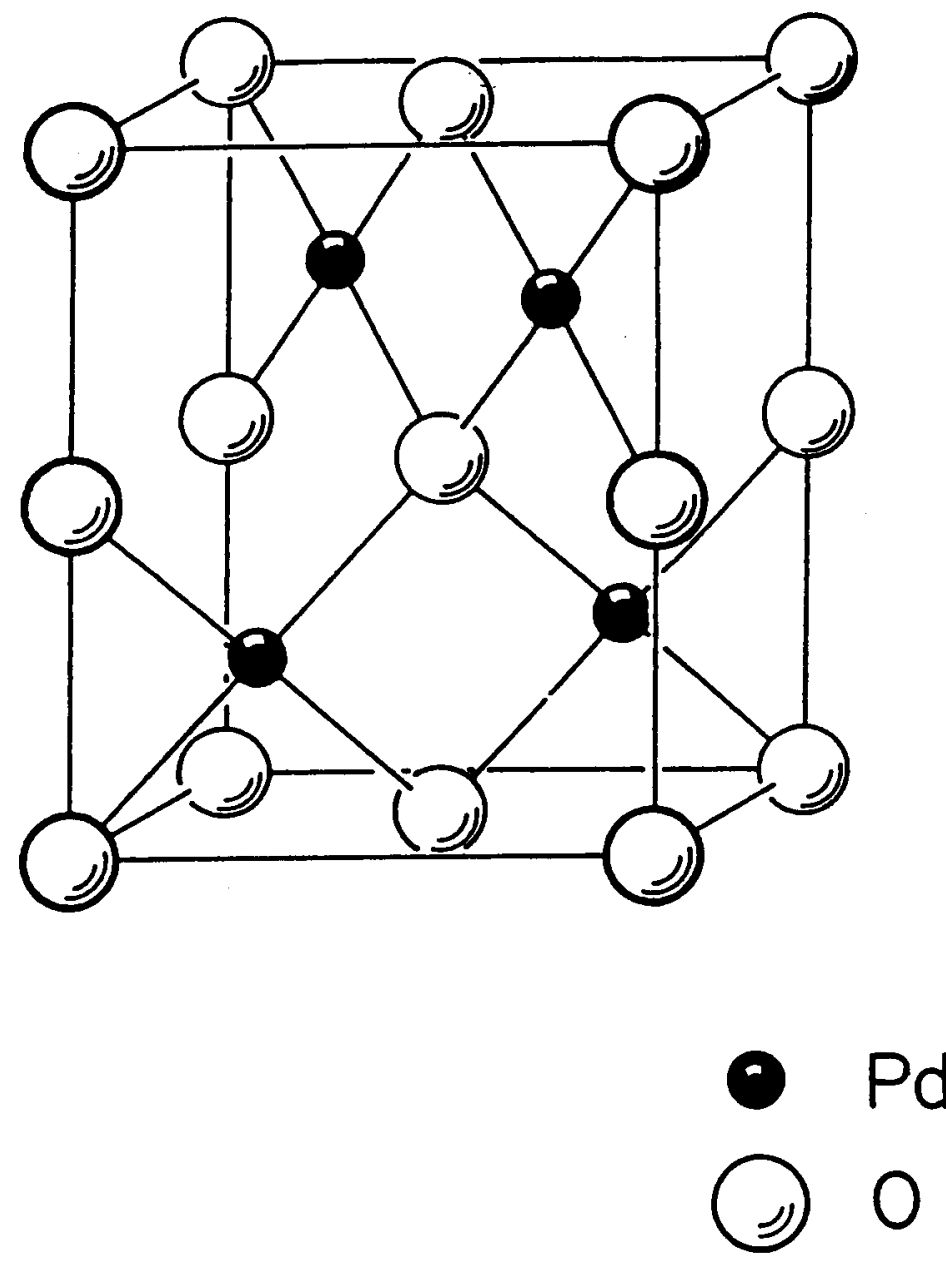

Figure 11: Archimedean Cooperite network as structure of $\mathrm{PdO}$ and $\mathrm{PtS}$, with the Wells point symbol of $\left(4^{2} 8^{4}\right)\left(4^{2} 8^{2}\right)$, and of space group symmetry $\left(\mathrm{P} 4_{2} / \mathrm{mmc}\right)$ 


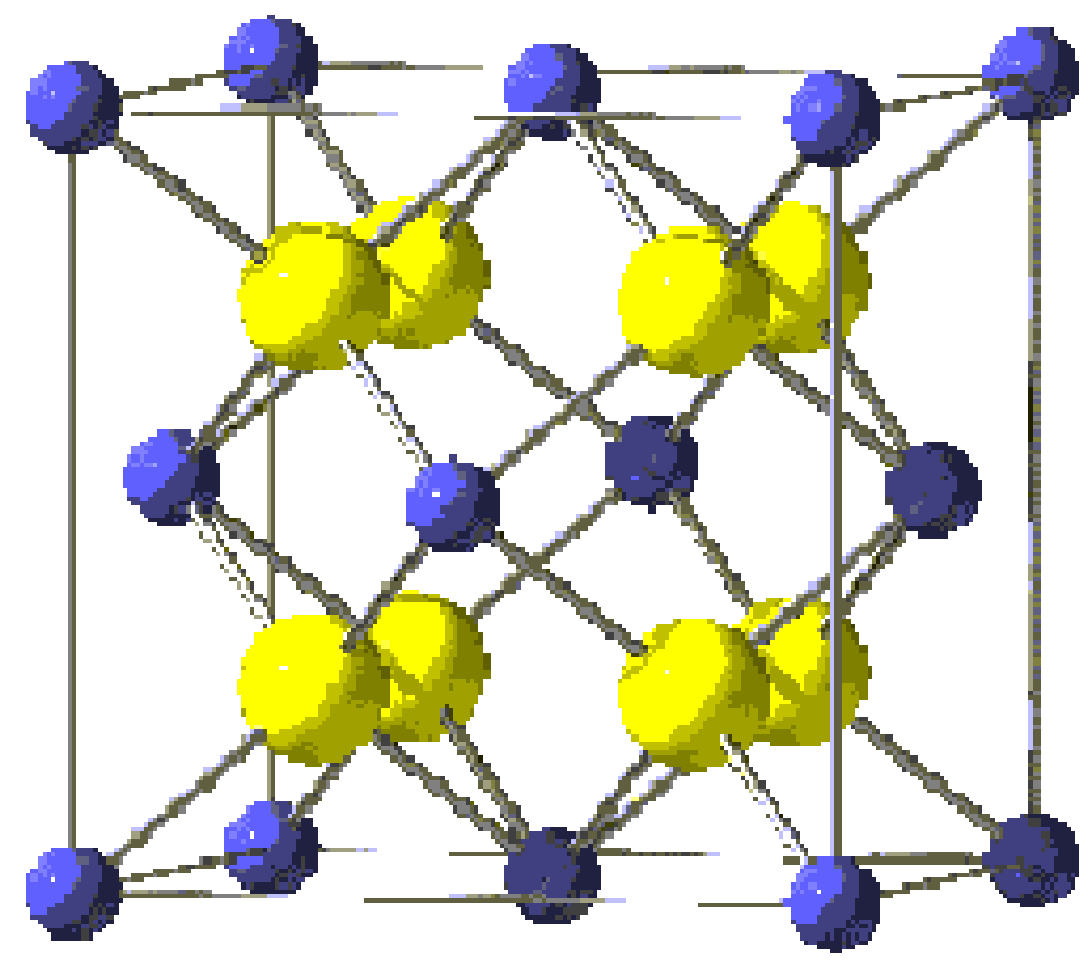

Figure 12: Catalan fluorite structure as the structure of $\mathrm{CaF}_{2}$, this network can be represented by the Wells point symbol $\left(4^{24}\right)\left(4^{6}\right)_{2}$, and lies in space group (Fm-3m) 


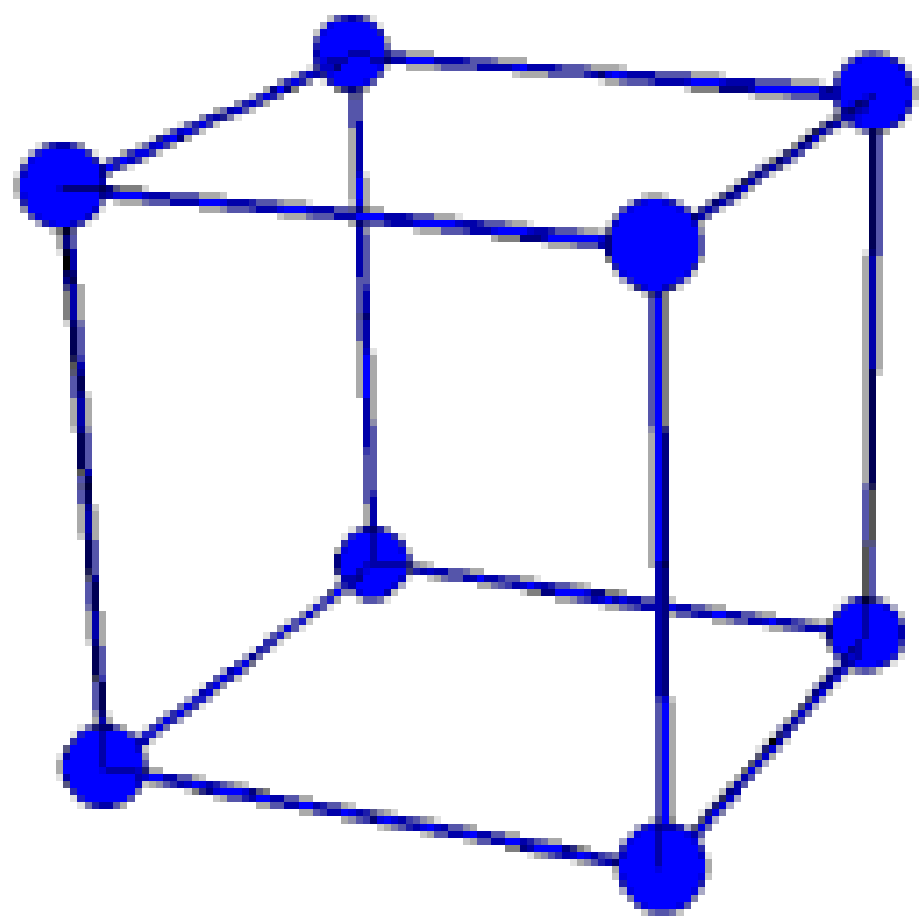

Figure 13: Platonic rocksalt structure as the structure of $\mathrm{NaCl}$, this network can be represented by the Wells point symbol $\left(4^{12}\right)$, and lies in space group $(\mathrm{Pm}-3 \mathrm{~m})$ 


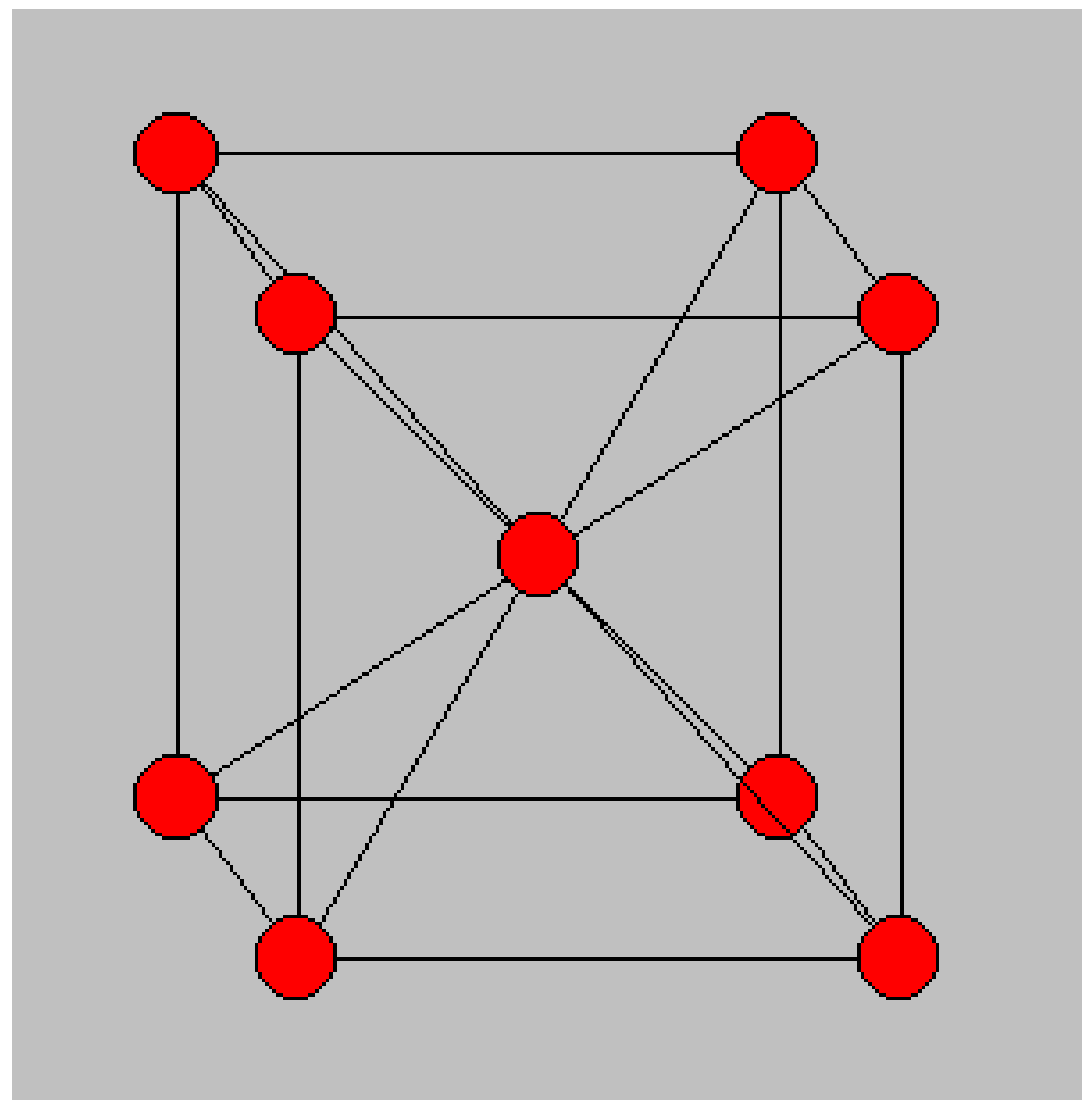

Figure 14: Platonic body-centered cubic (b.c.c.) structure as the structure of $\mathrm{CsCl}$, this network can be represented by the Wells point symbol $\left(4^{24}\right)$, and lies in space group (Im-3m) 


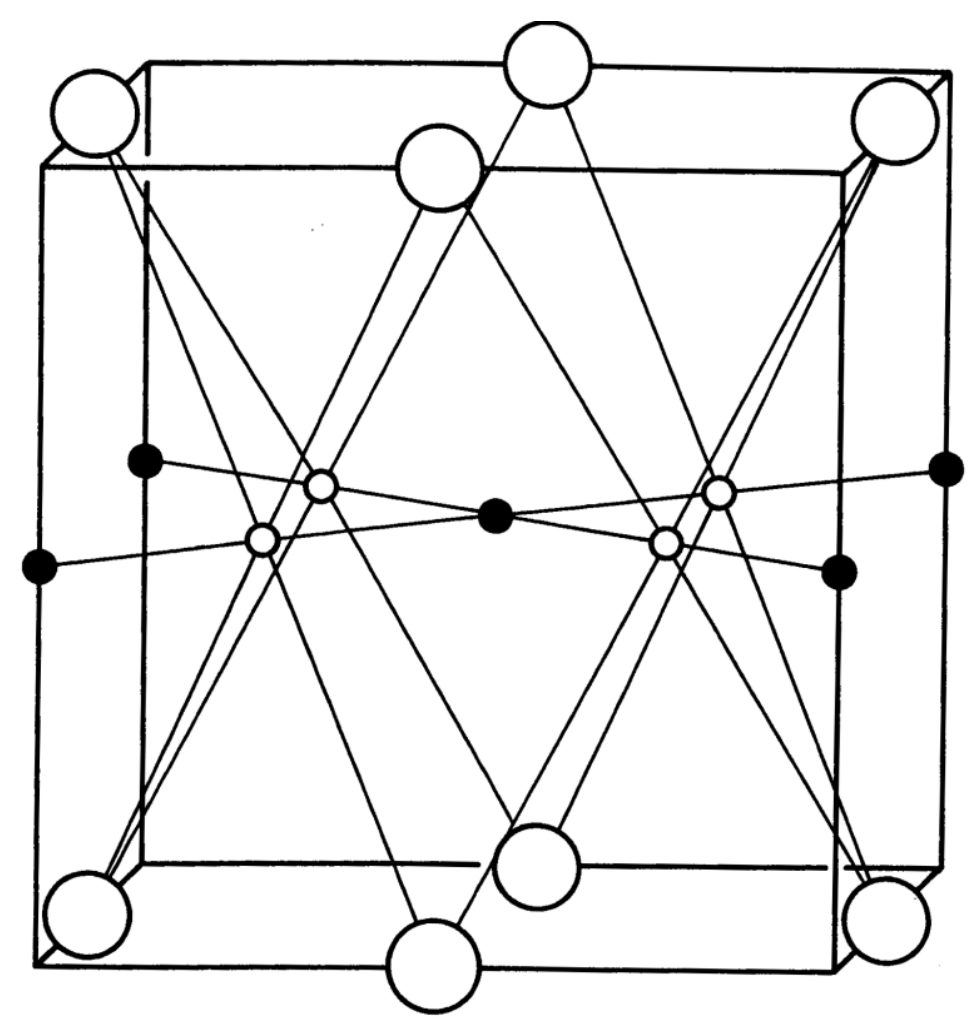

$$
\begin{aligned}
& \text { 8-connected (body centered) B } \\
& \text { 6-connected (octahedral) C } \\
& \text { 4-connected (square planar) A }
\end{aligned}
$$

Figure 15: Wellsean Kentuckia $\left(\mathrm{ABC}_{2}\right)$ structure-type as the structure of the superconducting cuprate salt $\mathrm{CaCuO}_{2}$, this network can be represented by the Wells point symbol $\left(4^{4}\right)\left(4^{12} 6^{12}\right)\left(4^{12}\right)_{2}$, and lies in space group (P4/mmm) 

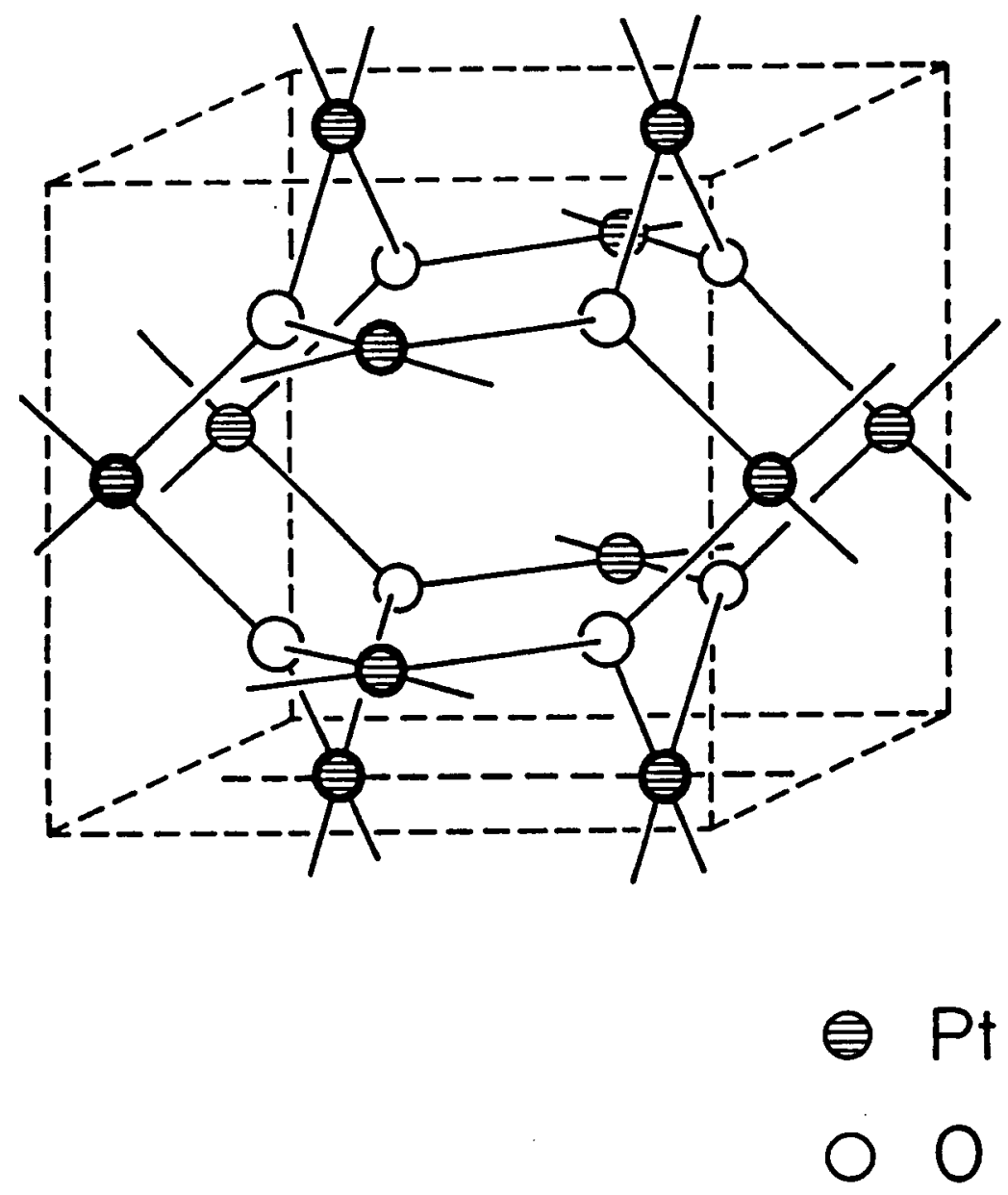

Figure 16: Catalan Waserite structure-type as the structure of the ionic conducting platinate salt $\mathrm{NaPt}_{3} \mathrm{O}_{4}$ (sodium cations not shown), this network can be represented by the Wells point symbol $\left(8^{4}\right)_{3}\left(8^{3}\right)_{4}$, and lies in space group (Pm-3n) 

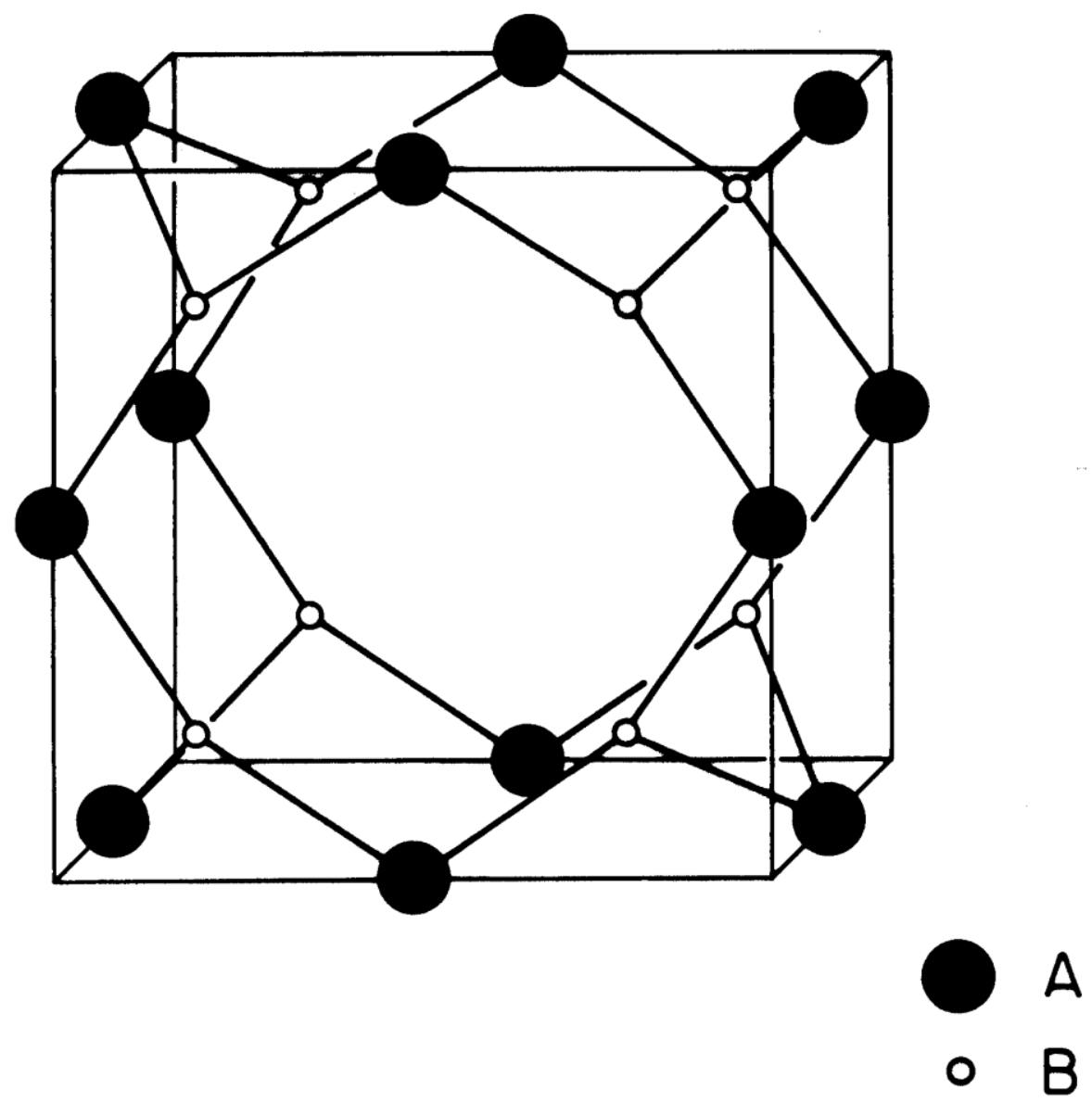

Figure 17: Wellsean Moravia structure-type as the structure of several coordination networks (metal organic frameworks, MOF's), this network can be represented by the Wells point symbol $\left(4^{4} 6^{8} 8^{12}\right)_{3}\left(4^{3}\right)_{8}$, and lies in space group (Pm-3m) 